\title{
THE NOTION OF VERTEX OPERATOR COALGEBRA AND A GEOMETRIC INTERPRETATION
}

\author{
KEITH HUBBARD
}

\begin{abstract}
The notion of vertex operator coalgebra is presented and motivated via the geometry of conformal field theory. Specifically, we describe the category of geometric vertex operator coalgebras, whose objects have comultiplicative structures meromorphically induced by conformal equivalence classes of worldsheets. We then show this category is isomorphism to the category of vertex operator coalgebras, which is defined in the language of formal algebra. The latter has several characteristics which give it the flavor of a coalgebra with respect to the structure of a vertex operator algebra and several characteristics that distinguish it from a standard dual - both will be mentioned.
\end{abstract}

\section{INTRODUCTION}

In this paper, we define the notion of a vertex operator coalgebra as motivated through the geometry of conformal field theory. Specifically, after defining the category whose objects are vertex operator coalgebras (VOCs) we show that this category is isomorphic to the category whose objects are geometrically defined structures called geometric vertex operator coalgebras (GVOCs). The results presented in this paper reflect a portion of the results proven in the author's Ph.D. dissertation Hub1. The definition of a geometric vertex operator coalgebra is motivated in a similar way to that of a geometric vertex operator algebra ([H1], [H2]) in that both arise from the geometry underlying conformal field theory, specifically closed string theory, in physics. Conceptually, string theory seeks to unify all the fundamental forces in nature into a single theory by modelling particles as one-dimensional objects (as opposed to point-particles) moving through spacetime. These objects, called strings, sweep out two-dimensional manifolds, called worldsheets, in space-time that model the interactions of particles.

Closed string theory focuses specifically on strings that begin and end at the same point, that is, on loops. Their study, along with the study of the algebraic structure they induce began in physics (cf. [BPZ, [FS, $[\underline{S}]$ and $[\underline{V}]$ ). Physicists introduced the notion of vertex operators in order to write down the expectation values of certain particle interactions. These vertex operators were independently discovered in mathematics in the study of representations of affine Lie algebras ([LW]). Shortly afterwards, Borcherds, attempting to describe the algebra arising from particle states and their vertex operators in a concise mathematical structure, introduced the notion of a vertex algebra $([\mathrm{BO}])$. This notion was then specialized in [FLM] to the notion of a vertex operator algebra (VOA). Although vertex operator algebras had origins in the geometry of string theory, a rigorous correspondence between the algebraic formalism and the geometric motivation was not completed

Date: November 6, 2018. 
until Huang introduced the notion of a geometric vertex operator algebra (GVOA) in [H1 and H2. Motivated by the physicists' study of the geometric interaction (specifically chiral interaction) of $n \in \mathbb{N}$ closed strings combining to form one closed string, Huang defined geometric vertex operator algebras in the context of a mathematical description of genus-zero Riemann surfaces with tubes up to conformal equivalence, along with a sewing operation on these objects. He also proved that the category of GVOAs was isomorphic to the category of VOAs, finalizing the rigorous correspondence between VOAs and the geometry of genus-zero worldsheets. The interactions corresponding to the worldsheets that Huang focused on are important special cases of interactions that may occur in a conformal field theory.

Another way that Huang's definition of GVOAs proved fruitful, was that it showed a VOA could be described in the language of operads, a language first developed by May in [M] to discuss iterated loop spaces. Specifically, Huang's GVOA corresponds to describing the partial operad of equivalence classes of genuszero compact Riemann surfaces with $n$ incoming punctures, one outgoing puncture and local coordinates vanishing at each puncture, then taking the meromorphic associative algebra associated to $\mathbb{C}$-extensions of that partial operad ([HL]). Using this interpretation, attaching or "sewing" two worldsheets together corresponds to composing operators in an operad defined in the category of complex vector spaces.

Prior to VOAs being solidly tied to their foundations in the geometry of conformal field theory, significant applications for VOAs had been emerging outside of conformal field theory. VOAs are now known to have deep connections to modular functions, have contributed to the representation theory of the Monster finite simple group and are fundamental to the study of representations of infinite-dimensional Lie algebras. (See the introduction of [FLM] for a more thorough exposition on connections and applications.)

Geometric VOAs are quite effective in modelling the interactions for which they were designed, specifically the combining of $n$ incoming strings into one outgoing string. There are, however, still very significant questions about what occurs in other settings. Geometric vertex operator coalgebras are designed to model genus-zero worldsheets corresponding to one incoming closed string splitting into $n$ outgoing closed strings. Similar to the GVOA case, this amounts to describing the partial operad of equivalence classes of genus-zero compact Riemann surfaces with one incoming and $n$ outgoing punctures, then investigating the algebraic structure it induces on complex vector spaces, i.e. determining the meaning of a coalgebra over this partial operad. The interactions modelled by GVOCs are of interest in their own right but also open the possibility of describing even more particle interactions when combined with existing GVOA theory. For instance, sewing the one incoming puncture of a worldsheet for a GVOC with the one outgoing puncture of a worldsheet for a GVOA, one obtains a worldsheet with any number of incoming and any number of outgoing punctures. Attaching the incoming punctures of a worldsheet associated to a GVOA with the outgoing punctures of a worldsheet associated to a GVOC forms a higher-genus worldsheet. Exploring the algebraic compositions of the two operators corresponding to these two worldsheets may shed light on understanding higher-genus conformal field theory, which has been an area of marked difficulty in the past. It is also significant to note that any full conformal field theory must include VOCs as well as VOAs. 
The main thrust of this paper, however, is to understand VOCs from an algebraic and geometric standpoint. To that end, the notion of a vertex operator coalgebra is defined (purely in terms of vector spaces, formal variables and formal operators) and is shown to be categorically isomorphic to the notion of GVOC. Generating a formal algebraic description in the VOA case has facilitated a more concrete understanding of the structure as well as the construction of examples. Similarly the algebraic description of VOCs allows us to describe concrete examples (Hub2]) and opens the door to a deeper understanding of a conformal field theory's algebraic structure. One might reasonably wonder whether the definition of VOC might be arrived at through purely algebraic means. But "standard" dualizing would not produce bounding of formal variables in opposite directions that arise in VOCs specifically, truncation produces only finitely many positive powers of the given formal variable in a VOC, while the counit property produces only non-negative powers (in VOAs everything is truncated from below). Additionally, results of weak commutativity coming from the VOC Jacobi identity reveal an important distinction from that of the VOA Jacobi identity (cf. Hub3]).

Since VOAs are of interest outside of string theory, it is natural to hope that VOCs, once well understood, might introduce new connections to broader mathematics as well.

In Section 2 we will recall Huang's conventions ([H1, H2 ) for spheres with multiple outgoing tubes and make the obvious generalizations of notation and theory to spheres with multiple incoming tubes. Section 3 will cover the necessary formal calculus to describe the context in which our comultiplication operators will be defined. In Section 4 we will define the notion of geometric vertex operator coalgebra as well as the notion of (algebraic) vertex operator coalgebra and discuss why finding a coalgebra structure is more subtle than the process of reversing the orientation of spheres with tubes (see Remark 4.4). There will also be a number of properties of VOCs presented in Section 4. Section 5 will provide a constructive isomorphism from the category of GVOCs to the category of VOCs as well as its inverse.

Acknowledgements This paper reflects a portion of the author's Ph.D. dissertation. The author would like to thank his advisor, Katrina Barron, for her guidance and encouragement throughout the research, writing and revising phases of this paper and his dissertation. In addition, he would like to express thanks to Stephan Stolz for numerous helpful conversations. The author also gratefully acknowledges the financial support of the Arthur J. Schmidt Foundation.

\section{The GEOMETRY OF SPHERES WITH TUBES}

We begin by investigating the geometry of spheres with tubes. Since our main motivation in this paper is studying closed strings propagating through space-time, it is essential that we precisely define the types of structures that model this behavior. The description in this section will closely follow $\mathrm{H} 2$ since that work contains the description of the geometry underlying GVOAs (motivated by string theory) and also because we would like the geometry of GVOCs to be compatible with that structure. 
2.1. Defining spheres with tubes. Geometric vertex operator algebras and coalgebras are defined using the moduli space (under conformal equivalence) of genuszero compact Riemann surfaces with punctures and local coordinates vanishing at each puncture. Vafa first observed that having a puncture on a Riemann surface together with local coordinates that vanish at that puncture is conformally equivalent to having a half-infinite tube attached to the Riemann surface (cf. $[\mathbf{V}],[\mathrm{H} 2]$ ). We will begin by defining exactly what we mean by a genus-zero Riemann surface with punctures and local coordinates vanishing at the punctures, or equivalently, a genus-zero worldsheet.

Consider a compact genus-zero Riemann surface, by which we mean a compact connected genus-zero one-dimensional complex manifold. In $\mathrm{H} 2$ and in this paper these surfaces are simply referred to as "spheres". The reason for the use of the word spheres is that any compact Riemann surface of genus-zero is complex analytically isomorphic to the standard Riemann sphere, i.e. $\mathbb{C} \cup\{\infty\}$ with the standard complex structure (cf. [A], FK]). Since we will eventually be concerned only with conformal equivalence classes of Riemann surfaces, in Section 2.3 we will pick canonical representatives of the equivalences classes under conformal equivalence and these canonical representatives will be Riemann spheres.

By the term oriented puncture we mean the selection of a point of a sphere together with an element of $\{+,-\}$. Given an oriented puncture $p$, a local analytic coordinate chart vanishing at $p$ is a pair $(U, \Phi)$ where $U$ is an open neighborhood of $p$ in the sphere, called the local coordinate neighborhood, and $\Phi: U \rightarrow \mathbb{C}$ is an injective analytic map, such that $\Phi(p)=0$, called the local coordinate map. The term tube centered at $p$ is used interchangeably with the term local analytic coordinate chart vanishing at $p$ and may by thought of as the path of a single closed string propagating through space-time. Tubes centered at negatively oriented punctures represent half-infinite tubes swept out by outgoing strings, while tubes centered at positively oriented punctures represent half-infinite tubes swept out by incoming strings.

Spheres with $m$ tubes centered at negatively oriented punctures and $n$ tubes centered at positively oriented punctures are said to be of type $(m, n)$. All oriented punctures are required to be distinct and have an ordering with negatively oriented punctures coming first. In [H2], the focus is primarily on spheres of type $(1, m)$, with $m \in \mathbb{N}$. In this work, we will discuss spheres of type $(1, m)$, for $m \in \mathbb{N}$, called spheres with incoming tubes, and of type $(m, 1)$ for $m \in \mathbb{N}$, called spheres with outgoing tubes, but will eventually focus on the latter.

For the moment, however, we will discuss all genus-zero worldsheets. Using the above framework, we can denote a sphere (or compact Riemann surface of genuszero) with $m$ outgoing punctures and $n$ incoming punctures by

$$
\left(S ; p_{-m}, \ldots, p_{-1}, p_{1}, \ldots, p_{n} ;\left(U_{-m}, \Phi_{-m}\right), \ldots,\left(U_{-1}, \Phi_{-1}\right),\left(U_{1}, \Phi_{1}\right), \ldots,\left(U_{n}, \Phi_{n}\right)\right)
$$

where $m, n \in \mathbb{N}, S$ is a compact genus-zero Riemann surface, $p_{i}$ is a point in $S$ with the sign of the index corresponding to the orientation of the puncture, and $\left(U_{i}, \Phi_{i}\right)$ is a local analytic coordinate chart vanishing at $p_{i}$ for $i=-m, \ldots-1,1, \ldots, n$. The terms sphere with tubes, genus-zero worldsheet, and compact Riemann surface of genus-zero with ordered punctures and local coordinates vanishing at the punctures are used interchangeably in the literature to refer to this structure. 
Let

$$
\begin{aligned}
\Sigma_{1}=\left(S_{1} ; p_{-m}, \ldots, p_{-1}, p_{1}, \ldots, p_{n} ;\right. \\
\left.\left(U_{-m}, \Phi_{-m}\right), \ldots,\left(U_{-1}, \Phi_{-1}\right),\left(U_{1}, \Phi_{1}\right), \ldots,\left(U_{n}, \Phi_{n}\right)\right)
\end{aligned}
$$

be a sphere of type $(m, n)$ and

$$
\begin{aligned}
\Sigma_{2}=\left(S_{2} ; q_{-k}, \ldots, q_{-1}, q_{1}, \ldots, q \ell ;\right. & \left.\left(V_{-k}, \Psi_{-k}\right), \ldots,\left(V_{-1}, \Psi_{-1}\right),\left(V_{1}, \Psi_{1}\right), \ldots,\left(V_{\ell}, \Psi_{\ell}\right)\right)
\end{aligned}
$$

be a sphere of type $(k, \ell)$. We say that $\Sigma_{1}$ and $\Sigma_{2}$ are conformally equivalent if $m=k, n=\ell$ and there is a complex analytic isomorphism $F: S_{1} \rightarrow S_{2}$ such that $F\left(p_{i}\right)=q_{i}$ and $\Phi_{i}=\Psi_{i} \circ F$ in some neighborhood of $p_{i}$ for $i=-m, \ldots,-1,1, \ldots, n$.

2.2. Sewing spheres with tubes. A fundamental property of the interactions of strings in conformal field theory is that certain interactions should compose naturally. In geometry we model composition by combining two Riemann surfaces into a single Riemann surface. This procedure is rigorously established in $\mathrm{H} 2$ for "sewing", or attaching, two spheres of type $(1, m)$ and $(1, n)$ respectively. However, Huang's description never uses the orientation on the punctures that remain unsown, allowing the argument to be directly generalized to sewing one incoming tube of a sphere of type $(m, n)$ to one outgoing tube of a sphere of type $(k, \ell)$ for $m, \ell \in \mathbb{N}, n, k \in \mathbb{Z}_{+}$. Following [H2], we will describe the conditions under which such a sewing may occur and describe the resulting sphere with tubes.

We will use $B^{r} \subset \mathbb{C}$ and $\bar{B}^{r} \subset \mathbb{C}$ to denote the open and closed discs of radius $r$ centered at the origin. With $\Sigma_{1}$ and $\Sigma_{2}$ as above, choose integers $1 \leq i \leq n$ and $1 \leq j \leq k$. We say that the $i$-th tube of $\Sigma_{1}$ can be sewn with the $-j$-th tube of $\Sigma_{2}$ if there exists $r \in \mathbb{R}_{+}$such that

$$
\begin{gathered}
\bar{B}^{r} \subset \Phi_{i}\left(U_{i}\right), \\
\bar{B}^{1 / r} \subset \Psi_{-j}\left(V_{-j}\right),
\end{gathered}
$$

$p_{i}$ is the only puncture in $\Phi_{i}^{-1}\left(\bar{B}^{r}\right)$, and $q_{-j}$ is the only puncture in $\Psi_{-j}^{-1}\left(\bar{B}^{1 / r}\right)$. Additionally, we say the $i$-th tube of $\Sigma_{1}$ can be sewn with the $-j$-th tube of $\Sigma_{2}$ if there exists $r \in \mathbb{R}_{+}$such that the domain of $\Phi_{i}^{-1}$ may be analytically extended to $\bar{B}^{r}$ without $\Phi_{i}^{-1}\left(\bar{B}^{r}\right)$ containing punctures other than $p_{i}$, and the domain of $\Psi_{-j}^{-1}$ may be analytically extended to $\bar{B}^{1 / r}$ without $\Psi_{-j}^{-1}\left(\bar{B}^{1 / r}\right)$ containing punctures other that $q_{-j}$. From such a $\Sigma_{1}$ and $\Sigma_{2}$ we obtain a sphere with tubes of type $(m+k-1, n+\ell-1)$ as follows. First, choose $r_{1}, r_{2}$ real numbers such that $0<r_{2}<r<r_{1},\left(\Phi_{i}\right.$ can be extended so that) $\bar{B}^{r_{1}} \subset \Phi_{i}\left(U_{i}\right),\left(\Psi_{-j}\right.$ can be extended so that) $\bar{B}^{1 / r_{2}} \subset \Psi_{-j}\left(V_{-j}\right), \Phi_{i}^{-1}\left(\bar{B}^{r_{1}}\right)$ contains no punctures of $\Sigma_{1}$ besides $p_{i}$, and $\Psi_{-j}^{-1}\left(\bar{B}^{1 / r_{2}}\right)$ contains no punctures of $\Sigma_{2}$ besides $q_{-j}$. Then define the sphere

$$
S_{3}=\left(\left(S_{1} \backslash \Phi_{i}^{-1}\left(\bar{B}^{r_{2}}\right) \sqcup\left(S_{2} \backslash \Psi_{-j}^{-1}\left(\bar{B}^{1 / r_{1}}\right)\right) / \sim\right.\right.
$$

where (using the notation of $[\underline{V}$ ) $\sqcup$ is the disjoint union and $\sim$ is the equivalence relation, preserving complex structure, given by $p \sim q$ if and only if $p=q$ or $p \in \Phi_{i}^{-1}\left(\bar{B}^{r_{1}}\right) \backslash \Phi_{i}^{-1}\left(\bar{B}^{r_{2}}\right), q \in \Psi_{-j}^{-1}\left(\bar{B}^{1 / r_{2}}\right) \backslash \Psi_{-j}^{-1}\left(\bar{B}^{1 / r_{1}}\right)$ and $\Psi_{-j}^{-1}\left(\frac{1}{\Phi_{i}(p)}\right)=q$. We 
define the sewing of the $i$-th tube of $\Sigma_{1}$ with the $-j$-th tube of $\Sigma_{2}$ to be the sphere $S_{3}$ with the ordered punctures

$$
q_{-k}, \ldots, q_{-j-1}, p_{-m}, \ldots, p_{-1}, q_{-j+1}, \ldots, q_{-1}, p_{1}, \ldots, p_{i-1}, q_{1}, \ldots, q_{\ell}, p_{i+1}, \ldots, p_{n}
$$

and the local coordinate maps restricted appropriately; that is, for each puncture $p$ of $\Sigma_{1}$ remove $\Phi_{i}^{-1}\left(\bar{B}_{1}^{r}\right)$ from the local coordinate neighborhood and restrict the local coordinate map at $p$ to this new local coordinate neighborhood, and do similarly for each puncture $q$ in $\Sigma_{2}$ and its local coordinate chart. This sewing is independent of the choice of $r, r_{1}, r_{2}$ ( $\left.\underline{\mathrm{H} 2}\right)$.

2.3. The moduli space of spheres with tubes. At first glance, spheres with tubes might appear to be exactly the right picture for modelling closed strings in space-time. However, a standard complex manifold structure turns out to be a bit too rigid for the suggested interactions in space-time. By definition, conformal field theories assume invariance of interactions under conformal transformations. Thus two spheres which possess an invertible conformal map between them will result in equivalent correlation functions for the resulting particle interaction.

First, we follow Huang's work ([H1], [H2]) describing canonical representatives for each conformal equivalence class of spheres of type $(1, n)$, for $n \in \mathbb{Z}_{+}$.

For any $r \in \mathbb{R}_{+}$and $z \in \mathbb{C}^{\times}$, let

$$
\begin{aligned}
& B_{0}^{r}=\{w \in \widehat{\mathbb{C}}|| w \mid<r\}, \\
& B_{z}^{r}=\{w \in \widehat{\mathbb{C}}|| z-w \mid<r\}, \\
& B_{\infty}^{r}=\{w \in \widehat{\mathbb{C}}|| 1 / w \mid<r\} .
\end{aligned}
$$

Recall that the reason we refer to any compact genus-zero Riemann surface as a sphere is that each one is complex analytically isomorphic to the standard sphere $\widehat{\mathbb{C}}=\mathbb{C} \cup\{\infty\}$. Furthermore, we have the following proposition.

Proposition 2.1. Any sphere with tubes of type $(1, n)$, for $n \in \mathbb{Z}_{+}$, is conformally equivalent to a sphere of the form

$$
\left(\widehat{\mathbb{C}} ; z_{-1}, z_{1}, \ldots, z_{n} ;\left(B_{z_{-1}}^{r_{-1}}, \Phi_{-1}\right),\left(B_{z_{1}}^{r_{1}}, \Phi_{1}\right),\left(B_{z_{2}}^{r_{2}}, \Phi_{2}\right), \ldots,\left(B_{z_{n}}^{r_{n}}, \Phi_{n}\right)\right)
$$

where $z_{-1}=\infty, z_{n}=0, z_{i} \in \mathbb{C}^{\times}$for $i=1, \ldots, n-1$, satisfying $z_{i} \neq z_{j}$ for $i \neq j, r_{i} \in \mathbb{R}_{+}$for $i=-1,1,2, \ldots, n$ and $\Phi_{-1}, \Phi_{1}, \Phi_{2}, \ldots, \Phi_{n}$ are analytic on $B_{z_{-1}}^{r_{-1}}, B_{z_{1}}^{r_{1}}, B_{z_{2}}^{r_{2}}, \ldots, B_{z_{n}}^{r_{n}}$, respectively, such that

$$
\begin{aligned}
\Phi_{i}\left(z_{i}\right) & =0 \quad i=-1,1, \ldots, n \\
\lim _{w \rightarrow \infty} w \Phi_{-1}(w) & =1, \\
\lim _{w \rightarrow z_{i}} \frac{\Phi_{i}(w)}{w-z_{i}} & \neq 0, \quad i=1, \ldots, n .
\end{aligned}
$$

This proposition is exactly what is proven in Proposition 1.3.1 of [H2].

Remark 2.2. In Huang's work, [H2], the outgoing puncture is labelled the 0-th puncture while the incoming punctures are labelled the first through $n$-th punctures. In order to generalize to multiple outgoing punctures, we refer to outgoing punctures with negative indices. For example, in Proposition 2.1 the lone outgoing puncture 
is referred to as $z_{-1}$ and its local coordinate chart is referred to as $\left(B_{z_{-1}}^{r_{-1}}, \Phi_{-1}\right)$ whereas these would be referred to as $z_{0}$ and $\left(B_{\infty}^{r_{0}}, \Phi_{0}\right)$, respectively, in $[\mathrm{H} 2$. Our new notation is required to consider multiple outgoing punctures but also highlights the natural symmetry between incoming and outgoing punctures.

The fact that every conformal equivalence class of spheres of type $(1, n)$, for $n \in \mathbb{Z}_{+}$, contains a sphere of the form (2.1) allows us to focus solely on the Riemann sphere, $\widehat{\mathbb{C}}$, with tubes and still include every equivalence class. The following proposition goes a step farther and describes the precise amount of information needed to specify a particular equivalence class.

Proposition 2.3. Let

$$
\begin{array}{r}
\Sigma_{1}=\left(\widehat{\mathbb{C}} ; \infty, z_{1}, \ldots, z_{n-1}, 0 ;\left(B_{\infty}^{r_{-1}}, \Phi_{-1}\right),\left(B_{z_{1}}^{r_{1}}, \Phi_{1}\right), \ldots,\right. \\
\left.\left(B_{z_{n-1}}^{r_{n-1}}, \Phi_{n-1}\right),\left(B_{0}^{r_{n}}, \Phi_{n}\right)\right)
\end{array}
$$

and

$$
\begin{array}{r}
\Sigma_{2}=\left(\widehat{\mathbb{C}} ; \infty, \zeta_{1}, \ldots, \zeta_{n-1}, 0 ;\left(B_{\infty}^{s_{-1}}, \Psi_{-1}\right),\left(B_{\zeta_{1}}^{s_{1}}, \Psi_{1}\right), \ldots\right. \\
\left.\left(B_{\zeta_{n-1}}^{s_{n-1}}, \Psi_{n-1}\right),\left(B_{0}^{s_{-n}}, \Psi_{-n}\right)\right)
\end{array}
$$

be two spheres of type $(1, n)$, for $n \in \mathbb{Z}_{+}$. Let $f_{-1}, f_{1}, \ldots, f_{n}$ and $g_{-1}, g_{1}, \ldots, g_{n}$ be the series obtained by expanding the analytic functions $\Phi_{-1}, \Phi_{1}, \ldots, \Phi_{n}$ and $\Psi_{-1}, \Psi_{1}, \ldots, \Psi_{n}$ around $w=\infty, z_{1}, \ldots, z_{n-1}, 0$ and $w=\infty, \zeta_{1}, \ldots, \zeta_{n-1}, 0$, respectively. The worldsheets $\Sigma_{1}$ and $\Sigma_{2}$ are conformally equivalent if and only if $z_{i}=\zeta_{i}$ for $i=1, \ldots, n-1$, and $f_{i}=g_{i}$ (as power series), for $i=-1,1, \ldots, n$.

This proposition along with its proof may by found in $\mathrm{H} 2$ (Proposition 1.3.3). To extend the above results to spheres of type $(1,0)$, we need the following two propositions (Propositions 1.3.4 and 1.3.6 in [H2], respectively).

Proposition 2.4. Any sphere with tubes of type $(0,1)$ is conformally equivalent to a sphere of the form

$$
\left(\widehat{\mathbb{C}} ; \infty ;\left(B_{\infty}^{r_{-1}}, \Phi_{-1}\right)\right)
$$

where $r_{1} \in \mathbb{R}_{+}$and $\Phi_{1}$ is an analytic function on $B_{\infty}^{r_{-1}}$ that can be expanded as

$$
\Phi_{1}(w)=\frac{1}{w}+\sum_{j=2}^{\infty} a_{j}\left(\frac{1}{w}\right)^{j+1}
$$

with each $a_{j} \in \mathbb{C}$.

Proposition 2.5. Two spheres of the form $Q_{1}=\left(\widehat{\mathbb{C}} ; \infty ;\left(B_{\infty}^{r_{-1}}, \Phi_{-1}\right)\right)$ and $Q_{2}=$ $\left(\widehat{\mathbb{C}} ; \infty ;\left(B_{\infty}^{s_{-1}}, \Psi_{-1}\right)\right)$ are conformally equivalent if and only if $f_{-1}=g_{-1}$ (as power series), where $f_{-1}$ and $g_{-1}$ are the power series expansions of $\Phi_{-1}$ and $\Psi_{-1}$, respectively, around $w=\infty$.

These propositions allow us to canonically describe conformal equivalence classes of spheres of type $(1, n)$ for $n \in \mathbb{N}$ as follows:

$$
\left(z_{1}, \ldots, z_{n-1} ; f_{-1}, f_{1}, \ldots, f_{n}\right) \quad \text { if } n>0
$$




$$
\left(f_{-1}\right) \quad \text { if } n=0
$$

where $z_{1}, \ldots, z_{n-1}$ are distinct nonzero complex numbers and $f_{-1}, f_{1}, \ldots, f_{n}$ are power series that are convergent in some positive neighborhood. These tuples represent the conformal equivalence classes of the worldsheets

$$
\begin{gathered}
\left(\widehat{\mathbb{C}} ; \infty, z_{1}, \ldots, z_{n-1}, 0 ;\left(B_{\infty}^{r_{-1}}, f_{-1}\right),\left(B_{z_{1}}^{r_{1}}, f_{1}\right),\right. \\
\left.\ldots,\left(B_{z_{n-1}}^{r_{n-1}}, f_{n-1}\right),\left(B_{0}^{r_{n}}, f_{n}\right)\right) \quad \text { if } n>0 \\
\left(\widehat{\mathbb{C}} ; \infty ;\left(B_{\infty}^{r_{-1}}, f_{-1}\right)\right) \quad \text { if } n=0,
\end{gathered}
$$

respectively, where $r_{-1}, r_{1}, \ldots, r_{n}$ are appropriately chosen radii of convergence so that the corresponding local coordinate maps are convergent within $B_{\infty}^{r_{-1}}, B_{z_{1}}^{r_{1}}, \ldots$, $B_{z_{n-1}}^{r_{n-1}}, B_{0}^{r_{n}}$, respectively, and the local coordinate neighborhoods do not overlap. Notice that the lack of specificity on the choice of the $r_{i}$ means that we are not choosing a specific canonical representative of each equivalence class in the most specific sense. This subtlety is usually suppressed and the above tuples are referred to as canonical representatives because the germ of each analytic function is the only data affecting which equivalence class a particular sphere with punctures belongs to.

We now turn our attention to spheres with outgoing tubes. The results for spheres of type $(n, 1)$, for $n \in \mathbb{Z}_{+}$follow directly from the spheres with incoming tubes case since the proofs of those propositions never refer to the orientations of punctures. In the case of spheres of type $(0,1)$, however, additional proof is necessary in order to normalize the lone incoming puncture at 0 instead of at $\infty$ as is the case for spheres of type $(1,0)$. (We normalize the single incoming puncture in the representative of conformal equivalence classes of spheres of type $(0,1)$ to be at 0 in order to maintain compatibility with the preexisting structure notation for GVOAs defined by Huang in [H2].)

Proposition 2.6. Any sphere with tubes of type $(n, 1)$, for $n \in \mathbb{Z}_{+}$is conformally equivalent to a sphere of the form

$$
\left(\widehat{\mathbb{C}} ; z_{-n}, \ldots, z_{-1}, z_{1} ;,\left(B_{z_{-n}}^{r_{-n}}, \Phi_{-n}\right), \ldots,\left(B_{z_{-1}}^{r_{-1}}, \Phi_{-1}\right),\left(B_{z_{1}}^{r_{1}}, \Phi_{1}\right)\right)
$$

where $z_{-n}=\infty, z_{1}=0, z_{i} \in \mathbb{C}^{\times}$for $i=-n+1, \ldots,-1$, satisfying $z_{i} \neq z_{j}$ for $i \neq j, r_{i} \in \mathbb{R}_{+}$for $i=-n, \ldots,-1,1$ and $\Phi_{-n}, \ldots, \Phi_{-1}, \Phi_{1}$ are analytic on $B_{z_{-n}}^{r_{-n}}, \ldots, B_{z_{-1}}^{r-1}, B_{z_{1}}^{r_{1}}$, respectively, such that

$$
\begin{aligned}
\Phi_{i}\left(z_{i}\right)=0 \quad & i=-n, \ldots,-1,1 \\
\lim _{w \rightarrow \infty} w \Phi_{-n}(w) & =1, \\
\lim _{w \rightarrow z_{i}} \frac{\Phi_{i}(w)}{w-z_{i}} & \neq 0, \quad i=-n+1, \ldots,-1,1 .
\end{aligned}
$$

Proof. The proof of Proposition 1.3.1 in [H2 suffices since it never uses the orientation of the punctures. 
As before, this proposition narrows the choices for a canonical representatives in each equivalence class of spheres of type $(n, 1)$ and the following proposition illuminates the exact minimum information required to reference a particular equivalence class.

Proposition 2.7. Let

$$
\begin{aligned}
& \Sigma_{1}=\left(\widehat{\mathbb{C}} ; \infty, z_{-n+1}, \ldots, z_{-1}, 0 ;\left(B_{\infty}^{r_{-n}}, \Phi_{-n}\right),\left(B_{z_{-n+1}}^{r_{-n+1}}, \Phi_{-n+1}\right), \ldots,\right. \\
& \left.\left(B_{z_{-1}}^{r_{-1}}, \Phi_{-1}\right),\left(B_{0}^{r_{1}}, \Phi_{1}\right)\right)
\end{aligned}
$$

and

$$
\begin{array}{r}
\Sigma_{2}=\left(\widehat{\mathbb{C}} ; \infty, \zeta_{-n+1}, \ldots, \zeta_{-1}, 0 ;\left(B_{\infty}^{s_{-n}}, \Psi_{-n}\right),\left(B_{\zeta_{-n+1}}^{s_{-n+1}}, \Psi_{-n+1}\right), \ldots,\right. \\
\left.\left(B_{\zeta_{-1}}^{s_{-1}}, \Psi_{-1}\right),\left(B_{0}^{s_{1}}, \Psi_{1}\right)\right)
\end{array}
$$

be two spheres of type $(n, 1)$, for $n \in \mathbb{Z}_{+}$. Let $f_{-n}, \ldots, f_{-1}, f_{1}$ and $g_{-n}, \ldots, g_{-1}, g_{1}$ be the series obtained by expanding the analytic functions $\Phi_{-n}, \ldots, \Phi_{-1}, \Phi_{1}$ and $\Psi_{-n}, \ldots, \Psi_{-1}, \Psi_{1}$ around $w=\infty, z_{-n+1}, \ldots, z_{-1}, 0$ and $w=\infty, \zeta_{-n+1}, \ldots, \zeta_{-1}, 0$, respectively. The worldsheets $\Sigma_{1}$ and $\Sigma_{2}$ are conformally equivalent if and only if $z_{i}=\zeta_{i}$, for $i=-n+1, \ldots,-1$ and $f_{i}=g_{i}$ (as power series), for $i=-n, \ldots,-1,1$.

Proof. Follows from Proposition 1.3.3 in $\underline{\mathrm{H} 2}$.

These two propositions allow us to choose a canonical representative for each conformal equivalence class of worldsheets of type $(n, 1)$ for $n \in \mathbb{Z}_{+}$. Here we switch notation slightly for power series centered at points other than 0 and $\infty$. Power series expanded thus far about 0, or about any nonzero complex number, have been power series in $w$, while the power series at $\infty$ had to be expanded in terms of $\frac{1}{w}$. However, for the nonzero complex punctures, expanding in terms of $\frac{1}{w}$ is also valid. In describing representatives for equivalence classes of spheres with outgoing tubes, we will use the latter convention for all outgoing (i.e., nonzero) punctures. Thus a canonical representative will be denoted

$$
\left(\frac{1}{z_{-n+1}}, \ldots, \frac{1}{z_{-1}} ; f_{-n}, \ldots, f_{-1}, f_{1}\right)
$$

where $z_{-n+1}, \ldots, z_{-1}$ are distinct nonzero complex numbers, $f_{-n} \ldots, f_{-1}$ are power series in $\frac{1}{w}$ centered at $z_{-n+1}, \ldots, z_{-1}$, respectively, that are convergent in some positive neighborhood, and $f_{1}$ is a power series in $w$ centered at 0 that is convergent in some positive neighborhood. This canonical representative will represent the equivalence class of worldsheets containing the worldsheet

$$
\begin{aligned}
&\left(\widehat{\mathbb{C}} ; \infty, \frac{1}{z_{-n+1}}, \ldots, \frac{1}{z_{-1}}, 0 ;\left(B_{\infty}^{r_{-n}}, f_{-n}\right),\left(B_{\frac{1}{z_{-n+1}}}^{r_{-n+1}}, f_{-n+1}\right),\right. \\
&\left.\ldots,\left(B_{\frac{1}{z_{-1}}}^{r_{-1}}, f_{-1}\right),\left(B_{0}^{r_{1}}, f_{1}\right)\right),
\end{aligned}
$$

where $r_{-n}, \ldots, r_{-1}, r_{1}$ are appropriately chosen radii of convergence so that each local coordinate map is convergent within its corresponding local coordinate neighborhood.

It turns out to be useful to refer to nonzero punctures as $\frac{1}{z}$, first because we will be applying the global transformation $w \mapsto \frac{1}{w}$ to the Riemann spheres used in [H2] 
in some sense (cf. Remark 2.17), second because it makes composing multiple shifts to $\infty$ clearer, and third because it simplifies the isomorphism between geometric vertex operator coalgebras and vertex operator coalgebras at the conclusion of this paper.

For spheres of type $(0,1)$ we use a similar approach to that used for spheres of type $(1,0)$ (studying linear fractional transformations of the sphere) as the following two propositions reveal. (For explicit proofs, see Hub1.)

Proposition 2.8. Any sphere with tubes of type $(0,1)$ is conformally equivalent to a sphere of the form

$$
\left(\widehat{\mathbb{C}} ; 0 ;\left(B_{0}^{r_{1}}, \Phi_{1}\right)\right)
$$

where $r_{1} \in \mathbb{R}_{+}$and $\Phi_{1}$ is an analytic function on $B_{0}^{r_{1}}$ that can be expanded as

$$
\Phi_{1}(w)=w+\sum_{j=2}^{\infty} a_{j} w^{j+1}
$$

for $a_{j} \in \mathbb{C}$.

Proceeding in the same way as we did in Proposition 2.5 for worldsheets of type $(1,0)$, we now argue:

Proposition 2.9. Two spheres of the form $Q_{1}=\left(\widehat{\mathbb{C}} ; 0 ;\left(B_{0}^{r_{1}}, \Phi_{1}\right)\right)$ and $Q_{2}=$ $\left(\widehat{\mathbb{C}} ; 0 ;\left(B_{0}^{s_{1}}, \Psi_{1}\right)\right)$ are conformally equivalent if and only if $f_{1}=g_{1}$ (as power series), where $f_{1}$ and $g_{1}$ are the power series expansions of $\Phi_{1}$ and $\Psi_{1}$, respectively, around $w=0$.

A representative for an equivalence class of spheres of type $(0,1)$ will be described as

which will represent the equivalence class of spheres of type $(1,0)$ containing

$$
\left(\widehat{\mathbb{C}} ; 0 ;\left(B_{0}^{r_{1}}, f_{1}\right)\right)
$$

where $r_{1}$ is an appropriately chosen radius of convergence.

Remark 2.10. Although we will be primarily concerned with the moduli space of spheres with incoming tubes and the moduli space of spheres with outgoing tubes separately, the above propositions allow us to describe the general moduli space of spheres of type $(m, n)$, for any $m, n \in \mathbb{N}$. The type $(0,0)$ case is a one element set (that is not sewable); the cases of spheres of types $(1,0)$ and $(0,1)$ have been described above; and given $m, n \in \mathbb{Z}_{+}$, the moduli spaces of spheres of type $(m, n)$ may be describes as

$$
\left(\frac{1}{z_{-m+1}}, \ldots, \frac{1}{z_{-1}}, z_{1}, \ldots, z_{n-1} ; f_{-m}, \ldots, f_{-1}, f_{1}, \ldots, f_{n}\right)
$$

by making the trivial generalization to Proposition 2.1 or [2.6 that the orientations of punctures other than the first and last are irrelevant. Here Equation (2.13) is the generalization of Equations (2.6) and (2.11), and should be understood similarly. 
2.4. Sewing moduli spaces of spheres with tubes. Now that we have described the moduli space of spheres with tubes (the moduli space of spheres with incoming tubes and the moduli space of spheres with outgoing tubes being subsets), we need to lift the sewing operation from the sewing of particular spheres to the sewing of elements of the moduli space. Given $\Sigma_{1}$ and $\Sigma_{2}$, two worldsheets such that the $j$-th outgoing puncture of $\Sigma_{2}$ may be sewn into the $i$-th incoming puncture of $\Sigma_{1}$ as in Section 2.2 let $\Sigma_{3}$ be the worldsheet resulting from the sewing. We need to be certain that given $\Sigma_{1}^{\prime}$ and $\Sigma_{2}^{\prime}$ in the same conformal equivalence classes as $\Sigma_{1}$ and $\Sigma_{2}$, respectively, that the $j$-th outgoing puncture of $\Sigma_{2}^{\prime}$ may be sewn into the $i$-th incoming puncture of $\Sigma_{1}^{\prime}$ and that the resulting sphere, $\Sigma_{3}^{\prime}$, is in the same equivalence class as $\Sigma_{3}$. But given complex analytic isomorphisms $G_{1}: \Sigma_{1} \rightarrow \Sigma_{1}^{\prime}$ and $G_{2}: \Sigma_{2} \rightarrow \Sigma_{2}^{\prime}$ witnessing conformal equivalence, a radius $r \in \mathbb{R}_{+}$that make $\Sigma_{1}$ and $\Sigma_{2}$ sewable is exactly one that makes $\Sigma_{1}^{\prime}$ and $\Sigma_{2}^{\prime}$ sewable. Further, $G_{1} \sqcup G_{2}$ will provide precisely the complex analytic isomorphism needed between $\Sigma_{3}$ and $\Sigma_{3}^{\prime}$.

When it is the case that two elements of the moduli space of spheres, $Q_{1}$ and $Q_{2}$, are sewable with the $j$-th outgoing tube of $\Sigma_{2}$ attaching to the $i$-th incoming tube of $\Sigma_{1}$, we denote the resulting sewing by $Q_{1} i_{-j} Q_{2}$ (cf. [V], [H2]). Now that we have established that this sewing operation, under appropriate conditions, is well-defined on elements of the moduli space of spheres with punctures, let

$$
\begin{aligned}
Q_{1} & =\left(z_{-m+1}^{-1}, \ldots, z_{-1}^{-1}, z_{1}, \ldots, z_{n-1} ; f_{-m}, \ldots, f_{-1}, f_{1}, \ldots, f_{n}\right) \\
Q_{2} & =\left(\zeta_{-k+1}^{-1}, \ldots, \zeta_{-1}^{-1}, \zeta_{1}, \ldots, \zeta_{\ell-1} ; g_{-k}, \ldots, g_{-1}, g_{1}, \ldots, g_{\ell}\right)
\end{aligned}
$$

be two elements of the moduli space with spheres such that the sewing $Q_{1}{ }_{i} \infty_{-j} Q_{2}$ exists. Equivalently we may require that given the canonical representatives of $Q_{1}$ and $Q_{2}$, for $\ell, m \in \mathbb{N}, k, n \in \mathbb{Z}_{+}$and $1 \leq i \leq n, 1 \leq j \leq k$, there exists $r>0$ with $f_{i}^{-1}\left(\bar{B}^{r}\right)$ and $g_{-j}^{-1}\left(\bar{B}^{1 / r}\right)$ well defined and containing only the punctures $z_{i}$ and $\zeta_{-j}^{-1}$, respectively (note that we consider $z_{n}=0$ and $\zeta_{-k}^{-1}=\infty$ ). In this case, there are $r_{1}$ and $r_{2}$ satisfying $0<r_{2}<r<r_{1}$ such that $f_{i}^{-1}\left(\bar{B}^{r_{1}}\right)$ and $g_{-j}^{-1}\left(\bar{B}^{1 / r_{2}}\right)$ are well defined and still contain only the punctures $z_{i}$ and $\zeta_{-j}^{-1}$, respectively. Choose

$$
Q_{3}=\left(\tilde{z}_{-m-k+2}^{-1}, \ldots, \tilde{z}_{-1}^{-1}, \tilde{z}_{1}, \ldots, \tilde{z}_{n+\ell-2} ; \tilde{f}_{-m-k+1}, \ldots, \tilde{f}_{-1}, \tilde{f}_{1}, \ldots, \tilde{f}_{n+\ell-1}\right)
$$

so that $Q_{1}{ }_{i} \infty_{-j} Q_{2}=Q_{3}$. Let $F$ be the unique conformal equivalence from the sewing of the canonical representatives of $Q_{1}$ and $Q_{2}$ to the canonical representative of $Q_{3}$. Since the sewing is in two components, there are also unique maps $F^{(1)}$ : $\widehat{\mathbb{C}} \backslash f_{i}^{-1}\left(\overline{B^{r_{2}}}\right) \rightarrow \widehat{\mathbb{C}}$ and $F^{(2)}: \widehat{\mathbb{C}} \backslash g_{-j}^{-1}\left(\overline{B^{1 / r_{1}}}\left\{\zeta_{-i}^{-1}\right\}\right) \rightarrow \widehat{\mathbb{C}}$ satisfying

$$
\begin{gathered}
\left.F^{(1)}(w)\right|_{f_{i}^{-1}\left(B^{\left.r_{2} \backslash \bar{B}^{r_{1}}\right)}\right.}=\left.F^{(2)}\left(g_{-j}^{-1}\left(\frac{1}{f_{i}(w)}\right)\right)\right|_{f_{i}^{-1}\left(B^{\left.r_{2} \backslash \bar{B}^{r_{1}}\right)}\right.} \\
F^{(1)}(\infty)=\infty \\
F^{(2)}(0)=0
\end{gathered}
$$




$$
\lim _{w \rightarrow \infty} \frac{F^{(1)}(w)}{w}=1
$$

We call (2.14) the sewing equation and (2.15), (2.16), (2.17) the normalization conditions. These are the same equations as developed in Section 1.4 of [H2] but in a slightly more general context. A good deal of work goes into describing the canonical representative of $Q_{1}{ }_{i} \infty_{-j} Q_{2}$ constructively, but this work is quite instructive and indeed necessary in several cases for the main isomorphism theorem between GVOCs and VOCs. We will include a pair of example of explicit sewing in Section 2.8

In nearly any structure with an operation, it is valuable to investigate what set together with the available operations generates all possible elements. That question is answered for the moduli space of spheres with incoming tubes in Proposition 1.3.9 of [H2] as follows.

Proposition 2.11. Any element of the moduli space of spheres of type $(1, n)$, for $n \in \mathbb{N}$, is generated under sewing by the element $\left(w^{-1}\right)$ of type $(1,0)$, elements of type $(1,1)$, and elements of type $(1,2)$ which are of the form $\left(z ; w^{-1}, w-z, w\right)$, for $z \in \mathbb{C}^{\times}$.

In the proposition $w^{-1}, w-z$, and $w$ should be understood as power series in $w^{-1}, w$, and $w$ centered at $\infty, z$ and 0 , respectively. We are able to demonstrate a similar generating set for the moduli space of spheres with outgoing tubes (using similar notation for power series).

Proposition 2.12. Any element $Q$ of the moduli space of spheres of type $(n, 1)$, for $n \in \mathbb{N}$, is generated under sewing by the element $(w)$ of type $(0,1)$, elements of type $(1,1)$, and elements of type $(2,1)$ which are of the form $\left(z^{-1} ; w^{-1}, w^{-1}-z, w\right)$, for $z \in \mathbb{C}^{\times}$.

Proof. The proof is analogous to the proof of Proposition 1.3.9 in H2, i.e., by using a Jordan curve to split a given sphere (cf. [Hub1).

2.5. Expansions in terms of infinitesimal local coordinates. Any local coordinate map at 0 may be expressed uniquely as

$$
\exp \left(\sum_{j \in \mathbb{Z}_{+}} A_{j} w^{j+1} \frac{d}{d w}\right) a_{0}^{w \frac{d}{d w}} w
$$

where the $A_{j} \in \mathbb{C}$ and $a_{0} \in \mathbb{C}^{\times}$. (See Proposition 2.1.1 in [H2] and the following discussion).

Any local coordinate map vanishing at $\infty$, on the other hand, may be written as

$$
\exp \left(-\sum_{j \in \mathbb{Z}_{+}} A_{j} w^{-j+1} \frac{d}{d w}\right) a_{0}^{-w \frac{d}{d w}} \frac{1}{w}
$$

where the $A_{j} \in \mathbb{C}$ and $a_{0} \in \mathbb{C}^{\times}$. (See Proposition 2.1.16 in [H2]). In Huang's treatment of these moduli spaces as well as in this work, the puncture at $\infty$ is usually normalized so that $a_{0}=1$. This is a direct consequence of the conditions 
(2.3) and (2.5) in the moduli space of spheres with incoming tubes and the condition (2.9) in the moduli space with outgoing tubes.

Any local coordinate map vanishing at a nonzero complex number, $z$, may be written as a local coordinate map at 0 composed with a shift of $z$ to 0 :

$$
\left.\exp \left(\sum_{j \in \mathbb{Z}_{+}} A_{j} x^{j+1} \frac{d}{d x}\right) a_{0}^{x \frac{d}{d x}} x\right|_{x=w-z},
$$

or as a local coordinate map at $\infty$ composed with a shift of $z$ to $\infty$ :

$$
\left.\exp \left(-\sum_{j \in \mathbb{Z}_{+}} A_{j} x^{-j+1} \frac{d}{d x}\right) a_{0}^{-x \frac{d}{d x}} \frac{1}{x}\right|_{x=\left(w^{-1}-z^{-1}\right)^{-1}} .
$$

We will use the expansion at 0 for incoming tubes (as in $\mathrm{H} 2$ ) and the expansion at $\infty$ for outgoing tubes. Let the sequence $A=\left\{A_{j}\right\}_{j \in \mathbb{Z}_{+}}$denote the higher order coefficients in a given exponential expansions in terms of the infinitesimal local coordinates, $x^{j+1} \frac{d}{d x}$, and let the notation $\left(a_{0},\left(A_{1}, A_{2}, \ldots\right)\right)$ or $\left(a_{0}, A\right)$ record all the coefficients in a given expansion. We will also use the notation $\mathbf{0}$ for the sequence of all zeros and $A(a)$ for $\left\{a^{j} A_{j}\right\}_{j \in \mathbb{Z}_{+}}$. When we are dealing with a sequence of formal variables, we will denote the sequence $\left(\alpha_{0},\left(\mathcal{A}_{1}, \mathcal{A}_{2}, \ldots\right)\right)$ or $\left(\alpha_{0}, \mathcal{A}\right)$ following [H2 and $[\mathrm{B}$.

Using this notation, the canonical representative for an equivalence class of genus-zero worldsheets of type $(1, n)$ can be expanded as

$$
\left(z_{1}, \ldots, z_{n-1} ; A^{(-1)},\left(a_{0}^{(1)}, A^{(1)}\right), \ldots,\left(a_{0}^{(n)}, A^{(n)}\right)\right),
$$

and the canonical representative for an equivalence class of type $(n, 1)$ can be expanded as

$$
\left(z_{-n+1}^{-1}, \ldots, z_{-1}^{-1} ; A^{(-n)},\left(a_{0}^{(-n+1)}, A^{(-n+1)}\right), \ldots,\left(a_{0}^{(-1)}, A^{(-1)}\right),\left(a_{0}^{(1)}, A^{(1)}\right)\right),
$$

with $n \in \mathbb{Z}_{+}$, the $z_{i}$ 's distinct nonzero complex numbers, $\left(a_{0}^{i}, A^{(i)}\right)$ recording the local coordinate map for the puncture of corresponding index, and for the first sequence in each list, $A^{(-1)}$ and $A^{(-n)}$ respectively, recording the higher order coefficients for the exponential expansion of the local coordinate map at $\infty$. As mentioned before, these local coordinate maps will always be normalized to have $a_{0}^{(-1)}=1$ and $a_{0}^{(-n)}=1$, respectively. Exponential notation also allows us to describe a canonical representative of type $(1,0)$ as

$$
\left(A^{(-1)}\right)
$$

where $A^{(-1)}$ records the coefficients of the exponential expansion at $\infty$, and to describe a canonical representative of type $(0,1)$ as

$$
\left(\left(1, A^{(1)}\right)\right)
$$

where $\left(1, A^{(1)}\right)$ records the local coordinate map for the puncture at 0 . We see from Proposition 2.4 that in the type $(1,0)$ case not only must $a_{0}^{(-1)}=1$ but $A_{1}^{(-1)}=0$ 
for all representatives. Also, Proposition 2.8 implies that in the type $(0,1)$ case $a_{0}^{(1)}=1$ and $A_{1}^{(1)}=0$.

2.6. A formal description of the moduli space of spheres with incoming and outgoing tubes. We begin by formalizing a notation for describing the moduli space of spheres with one outgoing and $n$ incoming tubes. For $n \in \mathbb{Z}_{+}$, let

$$
M^{n-1}=\left\{\left(z_{1}, z_{2}, \ldots z_{n-1}\right) \mid z_{i} \in \mathbb{C}^{\times}, z_{i} \neq z_{j} \text { for } i \neq j\right\}
$$

Note that for $n=1, M^{0}$ has exactly one element. We say that the series $\exp \left(\sum_{j \in \mathbb{Z}_{+}} A_{j} w^{j+1} \frac{d}{d w}\right) w$ is absolutely convergent in a neighborhood of 0 precisely if its expansion as powers of $w$ is absolutely convergent in a neighborhood of 0 . We then define

$$
\begin{aligned}
H=\left\{A=\left\{A_{j}\right\}_{j \in \mathbb{Z}_{+}} \in \mathbb{C}^{\infty}\right. & \mid \exp \left(\sum_{j \in \mathbb{Z}_{+}} A_{j} w^{j+1} \frac{d}{d w}\right) w \text { is absolutely } \\
& \text { convergent in some neighborhood of } 0\} .
\end{aligned}
$$

Proposition 2.13. The moduli space of spheres with tubes of type $(1, n)$, for $n \in$ $\mathbb{Z}_{+}$, can be identified with the set

$$
K(n)=M^{n-1} \times H \times\left(\mathbb{C}^{\times} \times H\right)^{n},
$$

and the moduli space of spheres with tubes of type $(1,0)$ can be identified with the set

$$
K(0)=\left\{A \in H \mid A_{1}=0\right\} .
$$

This is simply a restatement of Propositions 3.1.1 and 3.1.2 in H2 but is the obvious conclusion of Equations (2.20) and (2.22). Let

$$
K=\coprod_{n \in \mathbb{N}} K(n)
$$

be the moduli space of incoming tubes with spheres. Under the sewing operation (which corresponds to the ' ${ }^{\prime}$ ' notation of operads), $K$ is a partial operad (HL Section 5). The action of $\sigma \in S_{n}$ on $S$, a genus-zero Riemann surface with one outgoing and $n$ incoming punctures, is given by reordering the punctures and making the $i$-th incoming puncture the $\sigma(i)$-th incoming puncture for each $i=1, \ldots, n$. This action is invariant under conformal equivalence and, hence, induces an action of $\sigma$ on the moduli space of spheres of type $(1, n)$, that is $K(n)$.

Via Equations (2.21) and (2.23), we also establish a uniform way of describing a canonical representative of the moduli space of spheres with one incoming and $n$ ordered outgoing punctures.

Proposition 2.14. Let $\widetilde{M}^{n-1}=\left\{\left(\frac{1}{z_{-n+1}}, \ldots \frac{1}{z_{-1}}\right) \mid z_{i} \in \mathbb{C}^{\times}, z_{i} \neq z_{j}\right.$ for $\left.i \neq j\right\}$ and again let $H=\left\{A=\left\{A_{j}\right\}_{j \in \mathbb{Z}_{+}} \mid \exp \left(\sum_{j \in \mathbb{Z}_{+}} A_{j} w^{j+1} \frac{d}{d w}\right) w\right.$ is absolutely convergent in some neighborhood of 0$\}$. Then the moduli space of spheres with tubes of type $(n, 1)$, for $n \in \mathbb{Z}_{+}$, can be identified with the set 


$$
K^{*}(n)=\widetilde{M}^{n-1} \times H \times\left(\mathbb{C}^{\times} \times H\right)^{n},
$$

and the moduli space of spheres with tubes of type $(0,1)$ can be identified with the set

$$
K^{*}(0)=\left\{A \in H \mid A_{1}=0\right\} .
$$

This is simply the obvious interpretation of Equations (2.21) and (2.23). As in the case of $K$, we define

$$
K^{*}=\coprod_{n \in \mathbb{N}} K^{*}(n)
$$

to be the moduli space of spheres with outgoing tubes. Again, the sewing operation gives $K^{*}$ the structure of a partial operad. The action of $\sigma \in S_{n}$ on $K^{*}(n)$ is the same as that of $K$, induced from making the $i$-th outgoing puncture the $\sigma(i)$-th outgoing puncture for each $i=1, \ldots, n$ on any representative of the equivalence class acted upon. This action is invariant under conformal equivalence and, hence, induces an action of $\sigma$ on $K^{*}(n)$. See Hub1 for a more detailed discussion.

The next two propositions follow directly from observations on spheres with tubes and the fact that sewing and permutation are invariant under conformal equivalence. (They may also be thought of as extensions of the results of Huang on $K$ to $K^{*}$ by observing that orientation of punctures is not at issue.)

Proposition 2.15. Let $\ell, m \in \mathbb{N}$ and $n \in \mathbb{Z}_{+}$such that $m+n>1$. Choose $Q_{1} \in K^{*}(\ell), Q_{2} \in K^{*}(m), Q_{3} \in K^{*}(n)$ and integers $1 \leq i \leq m+n-1$ and $1 \leq j \leq n$. Then $Q_{1}{ }_{1} \infty_{-i}\left(Q_{2}{ }_{1} \infty_{-j} Q_{3}\right)$ exists if and only if one of the following 3 holds:

(1) $i<j, Q_{2}{ }_{1} \infty_{-j-\ell+1}\left(Q_{1}{ }_{1} \infty_{-i} Q_{3}\right)$ exists and

$$
Q_{1}{ }_{1} \infty_{-i}\left(Q_{2}{ }_{1} \infty_{-j} Q_{3}\right)=Q_{2}{ }_{1} \infty_{-j-\ell+1}\left(Q_{1}{ }_{1} \infty_{-i} Q_{3}\right)
$$

(2) $j \leq i<j+m,\left(Q_{1}{ }_{1} \infty_{-i+j-1} Q_{2}\right)_{1} \infty_{-j} Q_{3}$ exists and

$$
Q_{1}{ }_{1} \infty_{-i}\left(Q_{2}{ }_{1} \infty_{-j} Q_{3}\right)=\left(\begin{array}{lll}
Q_{1}{ }_{1} \infty_{-i+j-1} & Q_{2}
\end{array}\right)_{1} \infty_{-j} Q_{3}
$$

(3) $i \geq j+m, Q_{2}{ }_{1} \infty_{-j}\left(Q_{1}{ }_{1} \infty_{-i+m-1} Q_{3}\right)$ exists and

$$
Q_{1{ }_{1} \infty_{-i}}\left(Q_{2}{ }_{1} \infty_{-j} Q_{3}\right)=Q_{2}{ }_{1} \infty_{-j}\left(Q_{1}{ }_{1} \infty_{-i+m-1} Q_{3}\right) .
$$

Proposition 2.16. Let $Q_{1}, Q_{2} \in K^{*}(2), \sigma=(12) \in S_{2}$, and $\tau=(132) \in S_{3}$. Then $Q_{1}{ }_{1} \infty_{-1} Q_{2}$ exists if and only if $\tau\left(Q_{1}{ }_{1} \infty_{-2}\left(\sigma Q_{2}\right)\right)$ also exists. If this is the case,

$$
Q_{1}{ }_{1} \infty_{-1} Q_{2}=\tau\left(Q_{1}{ }_{1} \infty_{-2}\left(\sigma Q_{2}\right)\right) .
$$

Proposition 2.16 may be extended to all of the symmetric groups and spheres with outgoing tubes since every symmetric group is generated by transpositions and every sphere with outgoing tubes is generated as in Proposition 2.12

Remark 2.17. It is significant to note that $K$ and $K^{*}$ are isomorphic as partial operads. The isomorphism is given by the global transformation $I: \widehat{\mathbb{C}} \rightarrow \widehat{\mathbb{C}}: w \mapsto \frac{1}{w}$ on the underlying Riemann spheres of the canonical representatives of $K$ and $K^{*}$; in 
addition the isomorphism reverses orientation of punctures, changes each puncture location $z$ to $\frac{1}{z}$, and composes local coordinate maps with I. Finally, given $Q \in$ $K(n)$, with local coordinates at 0 given by $\left(a_{0}^{(n)}, A^{(n)}\right)$, renormalization via the map $\left(a_{0}^{(n)}\right)^{-w} \frac{d}{d w}$ is required to express $I(Q)$ in its canonical form, but the isomorphism is clearest when canonical representatives are not chosen. The transformation map is functorial on equivalence classes, i.e. it commutes with actions of the symmetric groups by construction and also commutes with the sewing operation. To see that I indeed commutes with sewing, note that given $S_{1}$ and $S_{2}$, two spheres with incoming tubes, the conditions for the sewing $S_{1}{ }_{i} \infty_{-1} S_{2}$ to exist are precisely the same conditions required for the sewing $I\left(S_{2}\right)_{1} \infty_{-i} I\left(S_{1}\right)$ to exist and, in fact, when both exist

$$
I\left(S_{1}{ }_{i} \infty_{-1} S_{2}\right)=I\left(S_{2}\right)_{1} \infty_{-i} I\left(S_{1}\right)
$$

It is also useful to recall that $K^{*}(1)$ and $K(1)$ are both names for equivalence classes under conformal isomorphism of spheres of type $(1,1)$, i.e. $K^{*}(1)$ and $K(1)$ are equal as sets with identical action of the Virasoro algebra, and identical compositions $K^{*}(1) \times K^{*}(1) \rightarrow K^{*}(1)$ and $K(1) \times K(1) \rightarrow K(1)$. (But note that $I$ is not the identity map between $K(1)$ and $K^{*}(1)$.)

2.7. Commutation in the Virasoro algebra. The main obstacle to describing the local coordinate maps of $Q_{1} 1_{1} \infty_{-i} Q_{2}$ in exponential notation is the fact that the operators $\left\{w^{j+1} \frac{d}{d w}\right\}_{j \in \mathbb{Z}}$ do not commute. This was overcome by a somewhat specialized approach in $[\mathrm{H} 2$ but since that time a more powerful method has been introduced in BHL which applies to the Baker-Cambell-Hausdorff formula in general. This result is applicable because the operators $\left\{-w^{j+1} \frac{d}{d w}\right\}_{j \in \mathbb{Z}}$ give a representation of the Virasoro algebra.

Let $\mathcal{V}=\oplus_{n \in \mathbb{Z}} \mathbb{C} L_{n} \oplus \mathbb{C} d$ be the Virasoro algebra with the usual commutation relations

$$
\begin{aligned}
{\left[L_{m}, L_{n}\right] } & =(m-n) L_{m+n}+\frac{m^{3}-m}{12} \delta_{m,-n} d, \\
{[\mathcal{V}, d] } & =0
\end{aligned}
$$

for $m, n \in \mathbb{Z}$. For commuting formal variables $\left\{\mathcal{A}_{k}\right\}_{k \in \mathbb{Z}_{+}},\left\{\mathcal{B}_{k}\right\}_{k \in \mathbb{Z}_{+}}, \alpha_{0}$ and $\beta_{0}$, by BHL there exist unique $\left\{\Psi_{k}\right\}_{k \in \mathbb{Z}}$ and $\Gamma$ such that

$$
\begin{aligned}
e^{-\sum_{j \in Z_{+}} \mathcal{A}_{j} L_{j}} \alpha_{0}^{-L_{0}} \beta_{0}^{-L_{0}} e^{-\sum_{j \in \mathbb{Z}_{+}} \mathcal{B}_{j} L_{-j}} & \\
& =e^{-\sum_{j \in Z_{+}} \Psi_{-j} L_{-j}}\left(\alpha_{0} \beta_{0}\right)^{L_{0}} e^{\Psi_{0} L_{0}} e^{-\sum_{j \in Z_{+}} \Psi_{j} L_{j}} e^{\Gamma d} .
\end{aligned}
$$

Given $Q_{1}$ and $Q_{2}$ such that an incoming puncture of $Q_{1}$ with local coordinates $\left(a_{0}, A\right)$ may be sewn with an outgoing puncture of $Q_{2}$ having local coordinates $\left(b_{0}, B\right)$, these local coordinates may be substituted into the right-hand side of (2.24) and the corresponding $\left\{\Psi_{k}\right\}_{k \in \mathbb{Z}}$ and $\Gamma$ converge.

Remark 2.18. In $\left[\mathrm{H2}\right.$, the convergence of $\left\{\Psi_{k}\right\}_{k \in \mathbb{Z}}$ and $\Gamma$ are shown for sewings of $K(1)$ and then extend naturally to all sewings in $K$. But $K(1)$ and $K^{*}(1)$ are one and the same (i.e. conformal equivalence classes of spheres of type $(1,1)$ ), so convergence of $\left\{\Psi_{k}\right\}_{k \in \mathbb{Z}}$ and $\Gamma$ for sewings of $K^{*}(1)$ is already shown and extends 
naturally to all of $K^{*}$. For the proof of convergence in $K(1)$, see Corollary 4.3.2, Lemma 5.2.1 and p. 123 in $\mathrm{H} 2$.

Because $\Gamma$ depends on $\mathcal{A}, \mathcal{B}, \alpha_{0}$, and $\beta_{0}$, we denote $\Gamma$ by $\Gamma\left(\mathcal{A}, \mathcal{B}, \alpha_{0} \beta_{0}\right)$ following the notational convention of H2. The definition of GVOA, as well as GVOC, may be interpreted as depending on the choice of a section of the determinant line bundle over each equivalence class of spheres with tubes, in which case $d$ may be represented by the complex value that determines the section and $L_{k}$ is represented by $-w^{k+1} \frac{d}{d w}$ for $k \in \mathbb{Z}$.

2.8. Examples of explicit sewing and the sewing identities. It is instructive to sew a few simple spheres together in order to observe the explicit local coordinates that are generated.

Example 2.19. Let

$$
\begin{gathered}
Q_{1}=\left(\mathbf{0},\left(a_{0}^{(1)}, A^{(1)}\right)\right)=\left(w^{-1}, f_{1}\right) \in K^{*}(1), \\
Q_{2}=\left(\zeta^{-1} ; \mathbf{0},(1, \mathbf{0}),(1, \mathbf{0})\right)=\left(\zeta^{-1} ; w^{-1}, w^{-1}-\zeta, w\right) \in K^{*}(2),
\end{gathered}
$$

such that $Q_{1} 1_{1} \infty_{-2} Q_{2}$ exists. The unique $F^{(1)}$ and $F^{(2)}$ satisfying (2.14) are

$$
\begin{aligned}
& F^{(1)}(w)=w \\
& F^{(2)}(w)=f_{1}^{-1}(w)=\left(a_{0}^{(1)}\right)^{-w \frac{d}{d w}} e^{-\sum_{j \in \infty} A_{j}^{(1)} w^{j+1} \frac{d}{d w}} w .
\end{aligned}
$$

Thus

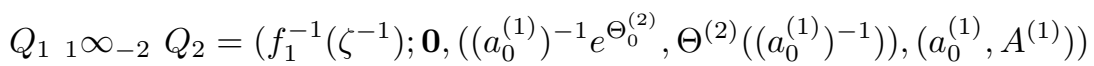

where $\Theta_{0}^{(2)}$ and $\Theta^{(2)}=\left\{\Theta_{j}^{(2)}\right\}_{j \in \mathbb{Z}_{+}}$are determined by

$$
\begin{aligned}
e^{-\sum_{j \in \mathbb{Z}_{+}}\left(a_{0}^{(1)}\right)^{-j} \Theta_{j}^{(2)} x^{-j+1} \frac{d}{d x}}\left(a_{0}^{(1)}\right)^{x \frac{d}{d x}} e^{-\left.\Theta_{0}^{(2)} x \frac{d}{d x} \frac{1}{x}\right|_{\frac{1}{x}=\frac{1}{w}-\frac{1}{f_{1}^{-1}(\zeta)}}}= \\
e^{\sum_{j \in \mathbb{Z}_{+}} A_{j}^{(1)} w^{j+1} \frac{d}{d w}} \frac{1}{w}-\zeta .
\end{aligned}
$$

Equivalently, let $\hat{f}_{2}(x)=e^{\sum_{j \in \mathbb{Z}}\left(a_{0}^{(1)}\right)^{-j} A_{j}^{(1)} x^{-j+1} \frac{d}{d x}} x$ so that

$$
Q_{1}{ }_{1} \infty_{-2} Q_{2}=\left(\left(a_{0}^{(1)} \hat{f}_{2}(\zeta)\right)^{-1} ; \mathbf{0},\left(\left(a_{0}^{(1)}\right)^{-1} e^{\Theta_{0}^{(2)}}, \Theta^{(2)}\left(\left(a_{0}^{(1)}\right)^{-1}\right)\right),\left(a_{0}^{(1)}, A^{(1)}\right)\right)
$$

where $\Theta_{0}^{(2)}$ and $\Theta^{(2)}=\left\{\Theta_{j}^{(2)}\right\}_{j \in \mathbb{Z}_{+}}$are determined by

$$
\left.e^{-\sum_{j \in \mathbb{Z}_{+}} \Theta_{j}^{(2)} x^{-j+1} \frac{d}{d x}} e^{-\Theta_{0}^{(2)} x \frac{d}{d x}} \frac{1}{x}\right|_{\frac{1}{x}=\frac{1}{w}-\hat{f}_{2}\left(\zeta^{-1}\right)}=\left(\hat{f}_{2}(w)\right)^{-1}-\zeta .
$$

Further, when considering formal variables $\alpha_{0}^{(1)}, \mathcal{A}^{(1)}$, and $\zeta$ instead of $a_{0}^{(1)}, A^{(1)}$, and $\zeta$, respectively, the $\Theta_{j}^{(2)}$ 's satisfy the sewing identity called the second sewing identity: 
Proposition 2.20. In the algebra $($ End $\mathbb{C})\left[w, w^{-1}\right][\zeta]\left[\alpha_{0}^{(1)}, \alpha_{0}^{(1)}\right]\left[\left[\mathcal{A}^{(1)}\right]\right]$, we have

$$
\begin{aligned}
& e^{-\sum_{k=-1}^{\infty}\left(\sum_{j \in \mathbb{Z}_{+}}\left(\alpha_{0}^{(1)}\right)^{-j} \mathcal{A}_{j}^{(1)}\left(\begin{array}{c}
-j+1 \\
k+1
\end{array}\right) \zeta^{-j-k}\right) w^{-k+1} \frac{\partial}{\partial w}} \\
& =e^{\Theta_{0}^{(2)} w \frac{\partial}{\partial w}} e^{\sum_{j \in \mathbb{Z}_{+}} \Theta_{j}^{(2)} w^{-j+1} \frac{\partial}{\partial w}} e^{\left(\zeta-\hat{f}_{2}(\zeta)\right) w^{2} \frac{\partial}{\partial w}} .
\end{aligned}
$$

Proof. This identity is observed from Proposition 2.2.9 of $\mathrm{H2}$ as follows. Let $x=w^{-1}, y=\zeta$, and $\mathcal{B}=\mathcal{A}^{(1)}\left(\left(\alpha_{0}^{(1)}\right)^{-1}\right)$ in Proposition 2.2.9, then take the inverse of both sides of the equation.

Using the second sewing equation, (2.26), we can generalize our example.

Example 2.21. Let

$$
\begin{gathered}
Q_{1}=\left(A^{(-1)},\left(a_{0}^{(1)}, A^{(1)}\right)\right) \in K^{*}(1), \\
Q_{2}=\left(\zeta^{-1} ; \mathbf{0},\left(b_{0}^{(-1)}, B^{(-1)}\right),(1, \mathbf{0})\right) \in K^{*}(2),
\end{gathered}
$$

such that $Q_{1}{ }_{1} \infty_{-2} Q_{2}$ exists. The unique $F^{(1)}$ and $F^{(2)}$ satisfying 2.14) are as in the previous example. Similarly, define $\hat{f}_{2}$ as above. Thus

$$
Q_{1}{ }_{1} \infty_{-2} Q_{2}=\left(\left(a_{0}^{(1)} \hat{f}_{2}(\zeta)\right)^{-1} ; A^{(-1)},\left(c_{0}^{(-1)}, C^{(-1)}\right),\left(a_{0}^{(1)}, A^{(1)}\right)\right)
$$

where $c_{0}^{(-1)}, C^{(-1)}=\left\{C_{j}^{(-1)}\right\}_{j \in \mathbb{Z}+}$ are defined by

$$
\begin{aligned}
& e^{-\sum_{j \in \mathbb{Z}_{+}} C_{j}^{(-1)} w^{-j+1} \frac{d}{d w}}\left(c_{0}^{(-1)}\right)^{-w \frac{d}{d w}} \\
= & e^{-\sum_{j \in \mathbb{Z}_{+}}\left(a_{0}^{(1)}\right)^{-j} \Theta_{j}^{(2)} w^{-j+1} \frac{d}{d w}}\left(\frac{e^{\Theta_{0}^{(2)}}}{a_{0}^{(1)}}\right)^{-w \frac{d}{d w}} e^{-\sum_{j \in \mathbb{Z}_{+}} B_{j}^{(-1)} w^{-j+1} \frac{d}{d w}}\left(b_{0}^{(-1)}\right)^{-w \frac{d}{d w}} .
\end{aligned}
$$

Note that $c_{0}^{(-1)}$ and $C^{(-1)}$ are uniquely determined (and independent of Virasoro representation) by $\mathrm{BHL}$.

We could also consider the sewing of $Q_{1}=\left(z^{-1} ; w^{-1}, w^{-1}-z, w\right) \in K^{*}(2)$ and $Q_{2}=\left(B^{(-1)},(1, \mathbf{0})\right) \in K^{*}(1)$ such that $Q_{1}{ }_{1} \infty_{-1} Q_{2}$ exists, and similarly achieve another sewing identity involving $\Theta_{j}^{(1)}$ 's and $\hat{f}_{1}(x)=e^{\sum_{j \in \mathbb{Z}} \mathcal{B}_{j}^{(-1)} x^{j+1} \frac{d}{d x}} x$ :

$$
\begin{aligned}
& e^{\left.\sum_{k=-1}^{\infty}\left(\sum_{j \in \mathbb{Z}_{+}} \begin{array}{c}
j+1 \\
k+1
\end{array}\right) \mathcal{B}_{j}^{(-1)} z^{j-k}\right) w^{-k+1} \frac{\partial}{\partial w}} \\
& =e^{\Theta_{0}^{(1)} w \frac{\partial}{\partial w}} e^{\sum_{j \in \mathbb{Z}_{+}} \Theta_{j}^{(1)} w^{-j+1} \frac{\partial}{\partial w}} e^{\left(z-\hat{f}_{2}^{-1}(z)\right) w^{2} \frac{\partial}{\partial w}} .
\end{aligned}
$$

(called the first sewing identity). This is done explicitly in Hub1 but directly follows the strategy we have used above.

2.9. The linear functionals $\mathcal{L}_{I}(z)$. In our work to understand representations of the Virasoro algebra in a given vertex operator coalgebra, a particular family of linear functionals will be of interest. First, we define the notion of a meromorphic function on any manifold $K^{*}(n)$, for $n \in \mathbb{N}$. 
Definition 2.22. A meromorphic function on $K^{*}(n)$ is a function that, when viewed as a function of $z_{-i}$, for $i=1, \ldots, n-1$, of $a_{0}^{(-i)}$, for $i=-1,1, \ldots, n-1$, and of $A_{j}^{(-i)}$, for $i=-1,1, \ldots, n$ and $j \in \mathbb{Z}_{+}$, is a polynomial in these variables divided by the product of powers of $a_{0}^{(-i)}$, for $i=-1,1, \ldots, n-1$, of $z_{-i}$, for $i=1, \ldots, n-1$, and of $z_{-i}-z_{-j}$, for $1 \leq i<j \leq n-1$ (cf. [H2] p. 67). Explicitly, if $F$ is a meromorphic function on $K^{*}(n)$ and $Q \in K^{*}(n)$ is as in 2.21) or (2.23), then

$$
F(Q)=\frac{\tilde{F}\left(z_{-n+1}, \ldots, z_{-1}, A^{(-n)}, \ldots, A^{(-1)}, A^{(1)}, a_{0}^{(-n+1)}, \ldots, a_{0}^{(-1)}, a_{0}^{(1)}\right)}{\left(a_{0}^{(1)}\right)^{r_{1}} \prod_{i=1}^{n-1}\left(a_{0}^{(-i)}\right)^{r_{-i}} \prod_{i<j}\left(z_{-i}-z_{-j}\right)^{s_{i j}} \prod_{i=1}^{n-1} z_{-i}^{t_{-i}}},
$$

where $\tilde{F}$ is a polynomial, and $r_{i}, s_{i j}, t_{i} \in \mathbb{N}$.

In addition to the definition, we will also use the following proposition which is Proposition 2.1.17 in $\underline{\mathrm{H} 2}$.

Proposition 2.23. Let $g(x) \in R\left[\left[x, x^{-1}\right]\right]$ and $f(x) \in R[[x]]$ with

$$
f(x)=e^{\sum_{k \in \mathbb{N}} \mathcal{A}_{k} x^{k+1} \frac{d}{d x}} x .
$$

Then if $g \circ f$ is well-defined,

$$
g \circ f(x)=e^{\sum_{k \in \mathbb{N}} \mathcal{A}_{k} x^{k+1} \frac{d}{d x}} g(x) .
$$

The linear functionals of interests will be denoted $\mathcal{L}_{I}(z), z \in \mathbb{C}^{\times}$, and will map from the space of meromorphic functions on $K^{*}(1)$ to $\mathbb{C}$. We define $\mathcal{L}_{I}(z)$ by

$$
\mathcal{L}_{I}(z) F=\left.\left(\frac{d}{d \varepsilon} F\left(((1,(0,-\varepsilon, 0,0, \ldots)))_{1} \infty_{-1}\left(z^{-1} ; \mathbf{0},(1, \mathbf{0}),(1, \mathbf{0})\right)\right)\right)\right|_{\varepsilon=0} .
$$

Since $\mathcal{L}_{I}(z)$ may be thought of as an element of the tangent space of $K^{*}(1)$ at $(\mathbf{0},(1, \mathbf{0}))$, it may be rewritten

$$
\mathcal{L}_{I}(z)=\left.\sum_{k \in \mathbb{Z}_{+}} \gamma_{-k} \frac{\partial}{\partial \mathcal{A}_{k}^{(-1)}}\right|_{\mathcal{A}^{(-1)}=\mathbf{0}}+\left.\gamma_{0} \frac{\partial}{\partial \alpha_{0}^{(1)}}\right|_{\alpha_{0}^{(1)}=1}+\left.\sum_{k \in \mathbb{Z}_{+}} \gamma_{k} \frac{\partial}{\partial \mathcal{A}_{k}^{(1)}}\right|_{\mathcal{A}^{(1)}=\mathbf{0}}
$$

for some coefficients $\gamma_{k}$, with $k \in \mathbb{Z}$. In fact, we can completely describe these coefficients as the following lemma shows.

Lemma 2.24 .

$\mathcal{L}_{I}(z)=\sum_{k \in \mathbb{Z}_{+}}-\left.z^{-k-2} \frac{\partial}{\partial \mathcal{A}_{k}^{(-1)}}\right|_{\mathcal{A}^{(-1)}=\mathbf{0}}-\left.z^{-2} \frac{\partial}{\partial \alpha_{0}^{(1)}}\right|_{\alpha_{0}^{(1)}=1}+\sum_{k \in \mathbb{Z}_{+}}-\left.z^{k-2} \frac{\partial}{\partial \mathcal{A}_{k}^{(1)}}\right|_{\mathcal{A}^{(1)}=\mathbf{0}}$

Proof. The idea of the proof will be to sew $((1,(0,-\varepsilon, 0,0, \ldots)))$ and $\left(z^{-1} ; \mathbf{0},(1, \mathbf{0}),(1, \mathbf{0})\right)$ together then examine what the derivative with respect to $\varepsilon$ at $\varepsilon=0$ is for each of the local coordinate coefficients. In some ways this reflects the approach in $\left[\mathrm{H} 2\right.$ to a similar linear functional $\mathcal{L}_{I}(z)$, but here we are forced to consider nontrivial scaling at the outgoing puncture and also the technique we use avoids the unresolved branching issue in (3.2.10) and (3.2.11) of [H2]. 
First, we denote the local coordinate map of $((1,(0,-\varepsilon, 0,0, \ldots)))$ as $f_{1}(w)$ and the local coordinate maps of $\left(z^{-1} ; \mathbf{0},(1, \mathbf{0}),(1, \mathbf{0})\right)$ as $g_{i}(w)$ where the subscript indicates which puncture the local coordinate map pertains to. Thus we get the sewing maps $F^{(1)}(w)=w$ and $F^{(2)}=f_{1}^{-1}\left(\frac{1}{g_{-1}(w)}\right)=e^{z w^{2} \frac{d}{d w}} e^{\varepsilon w^{3} \frac{d}{d w}} w$. These maps move the last outgoing puncture to $k=\left.e^{\varepsilon x^{3} \frac{d}{d x}} x\right|_{x=-z^{-1}}$. We employ the transformation map $T_{1}(w)=e^{k^{-1} w^{2} \frac{d}{d w}} w$. (Notice this does not guarantee that we will have a canonical representative of an element in $K^{*}(1)$ since there may be a nontrivial linear scaling of the local coordinate map at $\infty$.) After sewing and applying $T_{1}$, the local coordinate map at 0 is $g_{1} \circ\left(F^{(2)}\right)^{-1} \circ T_{1}^{-1}(w)$ and the local coordinate map at $\infty$ is $g_{-2} \circ\left(F^{(2)}\right)^{-1} \circ T_{1}^{-1}(w)$, both of which depend on $\varepsilon$ and will be trivial if $\varepsilon=0$.

Now, we take the derivative of the puncture at 0 with respect to $\varepsilon$ and evaluate at $\varepsilon=0$ (using Proposition 2.23 twice).

$$
\begin{aligned}
& \left.\frac{d}{d \varepsilon}\left(g_{1} \circ\left(F^{(2)}\right)^{-1} \circ T_{1}^{-1}(w)\right)\right|_{\varepsilon=0}=\left.\frac{d}{d \varepsilon}\left(e^{-k^{-1} w^{2} \frac{d}{d w}} e^{-\varepsilon w^{3} \frac{d}{d w}} e^{-z w^{2} \frac{d}{d w}} w\right)\right|_{\varepsilon=0} \\
& =\left.\frac{d}{d \varepsilon}\left(e^{-\left(-z^{-1}-\varepsilon z^{-3}-\ldots\right)^{-1} w^{2} \frac{d}{d w}}\left(\left(w^{-1}+z\right)^{-1}-\varepsilon w\left(w^{-1}+z\right)^{-2}+\ldots\right)\right)\right|_{\varepsilon=0} \\
& \quad=\frac{d}{d \varepsilon}\left(\left(\left(w^{-1}-\left(z^{-1}+\varepsilon z^{-3}+\ldots\right)^{-1}\right)+z\right)^{-1}-\varepsilon\left(w^{-1}-\right.\right. \\
& \left.\left.\left(z^{-1}+\varepsilon z^{-3}+\ldots\right)^{-1}\right)^{-1}\left(\left(w^{-1}-\left(z^{-1}+\varepsilon z^{-3}+\ldots\right)^{-1}\right)+z\right)^{-2}+\ldots\right)\left.\right|_{\varepsilon=0}
\end{aligned}
$$

We only use "..." for terms involving higher powers of $\varepsilon$, which will disappear upon differentiating and evaluating at $\varepsilon=0$, so this notation is unambiguous. Now applying the derivative and evaluating, the right-hand side of (2.29) at $\varepsilon=0$, we obtain

$$
\begin{gathered}
-\left(w^{-1}-z+z\right)^{-2} z^{2} z^{-3}-\left(w^{-1}-z\right)^{-1}\left(w^{-1}-z+z\right)^{-2} \\
=-w^{2} z^{-1}-w^{2}\left(w^{-1}-z\right)^{-1} \\
=-w^{2} \frac{z^{-1}}{1-w z}
\end{gathered}
$$

We are dealing with local coordinates at 0 which means there will be no negative powers of $w$. Thus

$$
\begin{gathered}
\left.\frac{d}{d \varepsilon}\left(g_{1} \circ\left(F^{(2)}\right)^{-1} \circ T_{1}^{-1}(w)\right)\right|_{\varepsilon=0}=-w^{2} \frac{z}{1-w z} \\
=-\sum_{k=0}^{\infty} z^{k-1} w^{k+2} .
\end{gathered}
$$

Using the same approach for the local coordinates at $\infty$, we find that

$$
\begin{gathered}
\left.\frac{d}{d \varepsilon}\left(g_{-2} \circ\left(F^{(2)}\right)^{-1} \circ T_{1}^{-1}(w)\right)\right|_{\varepsilon=0}=\left.\frac{d}{d \varepsilon}\left(\frac{1}{\left(F^{(2)}\right)^{-1} \circ T_{1}^{-1}(w)}\right)\right|_{\varepsilon=0} \\
=-\left.\left(\left(F^{(2)}\right)^{-1} \circ T_{1}^{-1}(w)\right)^{-2} \frac{d}{d \varepsilon}\left(\left(F^{(2)}\right)^{-1} \circ T_{1}^{-1}(w)\right)\right|_{\varepsilon=0}
\end{gathered}
$$




$$
\begin{gathered}
=-w^{-2}\left(-w^{2} \frac{z^{-1}}{1-w z}\right) \\
=-\frac{w^{-1} z^{-2}}{1-w^{-1} z^{-1}} \\
=-\sum_{k=0}^{\infty} z^{-k-2} w^{-k-1} .
\end{gathered}
$$

We see now that the local coordinates at $\infty$ do have an $a_{0}$ term that depends on $\varepsilon$ since their derivative evaluated at $\varepsilon=0$ has a nontrivial coefficient of $w^{-1}$ in the power series expansion, and we also see that since the coefficient of $w$ in the power series expansion is 0 , the local coordinates at 0 have a trivial $a_{0}$ term. We want to find out what the coefficients are in front of the canonical tangent vectors, though, so let $h_{1}(w)=e^{\sum_{k \in \mathbb{Z}_{+}} A_{k}^{(1)}(\varepsilon) w^{k+1} \frac{d}{d w}}\left(a_{0}^{(1)}(\varepsilon)\right)^{w} \frac{d}{d w} w$ and $h_{-1}(w)=$ $e^{\sum_{k \in \mathbb{Z}_{+}}-A_{k}^{(-1)}(\varepsilon) w^{-k+1} \frac{d}{d w}} \frac{1}{w}$ be the canonical representatives of the local coordinates obtained from the sewing (where $A_{k}^{(i)}(\varepsilon), a_{0}^{(1)}(\varepsilon)$ are functions depending on $\varepsilon$ for $i=$ $-1,1$ and $\left.k \in \mathbb{Z}_{+}\right)$. By applying the transformation map $T_{2}(w)=\left(a_{0}^{(1)}(\varepsilon)\right)^{w} \frac{d}{d w} w$, we again obtain the unique local coordinates with no nontrivial $a_{0}$ at 0 and a possibly nontrivial $a_{0}$ term at $\infty$. Taking the derivative of these local coordinates with respect to $\varepsilon$ at $\varepsilon=0$ yields

$$
\left.\frac{d}{d \varepsilon}\left(h_{1} \circ T_{2}^{-1}(w)\right)\right|_{\varepsilon=0}=\left.\sum_{k \in \mathbb{Z}_{+}}\left(\frac{d}{d \varepsilon} A_{k}^{(1)}(\varepsilon)\right)\right|_{\varepsilon=0} w^{k+1}
$$

for the puncture at 0 and

$$
\begin{gathered}
\left.\frac{d}{d \varepsilon}\left(h_{-1} \circ T_{2}^{-1}(w)\right)\right|_{\varepsilon=0}=\left.\frac{d}{d \varepsilon}\left(\left(a_{0}^{(1)}(\varepsilon)\right)^{-w \frac{d}{d w}} e^{-\sum_{k \in \mathbb{Z}_{+}} A_{k}^{(-1)}(\varepsilon) w^{-k+1} \frac{d}{d w}} \frac{1}{w}\right)\right|_{\varepsilon=0} \\
=\left.\left(\frac{d}{d \varepsilon}\left(a_{0}^{(1)}(\varepsilon)\right)\right)\right|_{\varepsilon=0} w^{-1}+\left.\sum_{k \in \mathbb{Z}_{+}}\left(\frac{d}{d \varepsilon} A_{k}^{(-1)}(\varepsilon)\right)\right|_{\varepsilon=0} w^{-k-1}
\end{gathered}
$$

for the puncture at $\infty$.

By comparing the coefficients of (2.30) and (2.32) as well as (2.31) and (2.33), we verify the claim of the lemma.

\section{Algebraic PReliminaries}

It is a standard notion in the theory of operads to study the formal algebraic structure that a geometric structure induces on vector spaces (cf. M], MSS and (HL). In order to study the kind of structure that $K^{*}$ induces on vector spaces, it is necessary to define and investigate some algebraic preliminaries. This section will cover the formal calculus we will need. For a more thorough exposition on formal calculus itself, see [FLM], FHL] or [LL]. 
3.1. The $\delta$-function. We will use the "formal $\delta$-function", $\delta(x)=\sum_{n \in \mathbb{Z}} x^{n}$, which is discussed in, for instance, FHL. Note that the $\delta$-function applied to $\frac{x_{1}-x_{2}}{x_{0}}$, where $x_{0}, x_{1}$ and $x_{2}$ are commuting formal variables, is a formal power series in $x_{2}$ (i.e., negative powers of $\left(x_{1}-x_{2}\right)$ are expanded in nonnegative integral powers of $\left.x_{2}\right)$. In general, in the formal calculus of VOAs the sum or difference of two formal variables, $\left(x_{1} \pm x_{2}\right)$, is understood to be expanded in nonnegative integral powers of the second, $x_{2}$ (cf. FLM], FHL).

The following three properties of $\delta$-functions will be relevant:

First, given a formal Laurent series $X\left(x_{1}, x_{2}\right) \in \operatorname{Hom}(V, W)\left[\left[x_{1}, x_{1}^{-1}, x_{2}, x_{2}^{-1}\right]\right]$ with coefficients which are homomorphisms from a vector space $V$ to a vector space $W$, if $\lim _{x_{1} \rightarrow x_{2}} X\left(x_{1}, x_{2}\right)$ exists (i.e. when $X\left(x_{1}, x_{2}\right)$ is applied to any element of $V$, setting $x_{1}=x_{2}$ leads to only finite sums in $W$ ) we have

$$
\delta\left(\frac{x_{1}}{x_{2}}\right) X\left(x_{1}, x_{2}\right)=\delta\left(\frac{x_{1}}{x_{2}}\right) X\left(x_{2}, x_{2}\right) .
$$

Second, we use the fact that

$$
x_{1}^{-1} \delta\left(\frac{x_{2}+x_{0}}{x_{1}}\right)=x_{2}^{-1} \delta\left(\frac{x_{1}-x_{0}}{x_{2}}\right)
$$

which is proved by direct expansion. The third fact may be observed by direct expansion and comparing of coefficients:

$$
x_{0}^{-1} \delta\left(\frac{x_{1}-x_{2}}{x_{0}}\right)-x_{0}^{-1} \delta\left(\frac{x_{2}-x_{1}}{-x_{0}}\right)=x_{2}^{-1} \delta\left(\frac{x_{1}-x_{0}}{x_{2}}\right) .
$$

3.2. Linear algebra on $\mathbb{Z}$-graded vector spaces with finite-dimensional homogeneous subspaces. Let $V=\coprod_{n \in \mathbb{Z}} V_{(n)}$ be a $\mathbb{Z}$-graded vector space over $\mathbb{C}$ such that $\operatorname{dim} V_{(n)}<\infty$, for each $n \in \mathbb{Z}$. We denote the graded dual space of $V$ by

$$
V^{\prime}=\coprod_{n \in \mathbb{Z}} V_{(n)}^{*}
$$

the algebraic closure of $V$ by

$$
\bar{V}=\prod_{n \in \mathbb{Z}} V_{(n)}=\left(V^{\prime}\right)^{*},
$$

and the natural pairing of $V^{\prime}$ with $\bar{V}$ by $\langle\cdot, \cdot\rangle$. The $n$-th tensor product of $V$, denoted $V^{\otimes n}$, is still a $\mathbb{Z}$-graded vector space (where $v \in V_{\left(k_{1}\right)} \otimes \ldots \otimes V_{\left(k_{n}\right)}$ has weight $\left.k_{1}+\ldots+k_{n}\right)$ with finite-dimensional homogeneous subspaces. Thus $\left(V^{\otimes n}\right)^{\prime}$, $\overline{V^{\otimes n}}$ and $\langle\cdot, \cdot\rangle:\left(V^{\otimes n}\right)^{\prime} \times \overline{V^{\otimes n}} \rightarrow \mathbb{C}$ are defined as above.

We denote a homogeneous basis of $V$ by

$$
\left\{e_{\ell^{(k)}}^{(k)} \mid k \in \mathbb{Z}, \ell^{(k)}=1, \ldots, \operatorname{dim} V_{(k)}\right\}
$$

and its corresponding dual basis of $V^{\prime}$ by

We will use the notation

$$
\left\{\left(e_{\ell^{(k)}}^{(k)}\right)^{*} \mid k \in \mathbb{Z}, \ell^{(k)}=1, \ldots, \operatorname{dim} V_{(k)}\right\} .
$$




$$
\mathcal{H}_{V}(m, n)=\operatorname{Hom}\left(V^{\otimes m}, \overline{V^{\otimes n}}\right)
$$

for $m, n \in \mathbb{N}$. For $m \in \mathbb{N}, n \in \mathbb{Z}_{+}$and any integer $0<i \leq n$, we define the t-contraction

$$
\begin{aligned}
\left(\cdot{ }_{1} *_{-i} \cdot\right)_{t}: \mathcal{H}_{V}(1, m) \times \mathcal{H}_{V}(1, n) & \rightarrow \operatorname{Hom}\left(V \overline{V^{\otimes m+n-1}}\left[\left[t, t^{-1}\right]\right]\right) \\
(f, g) & \mapsto\left(f_{1} *_{-i} g\right)_{t}
\end{aligned}
$$

where

$$
\begin{aligned}
&\left(f_{1} *_{-i}\right.g)_{t} v=\sum_{k \in \mathbb{Z}} \sum_{\ell^{(k)}=1}^{\operatorname{dim} V_{(k)}}(\underbrace{I d_{V} \otimes \cdots \otimes I d_{V}}_{i-1} \otimes f\left(e_{\ell^{(k)}}^{(k)}\right) \cdot\left(e_{\ell^{(k)}}^{(k)}\right)^{*} \otimes \\
&\underbrace{I d_{V} \otimes \cdots \otimes I d_{V}}_{n-i}) g(v) t^{k} .
\end{aligned}
$$

If for all $v^{\prime} \in\left(V^{\otimes n+m-1}\right)^{\prime}, v \in V$ the formal Laurent series

$$
\left\langle v^{\prime},\left(f_{1} *_{-i} g\right)_{t} v\right\rangle
$$

is absolutely convergent when $t=1$, then $\left(f_{1} *_{-i} g\right)_{1}$ is well-defined as an element of $\mathcal{H}_{V}(1, m+n-1)$ and we define the contraction of $f$ and $g$ by,

$$
f_{1} *_{-i} g=\left(f_{1} *_{-i} g\right)_{1} .
$$

The following associativity of $t$-contractions follows from the definition.

Proposition 3.1. Let $\ell, m \in \mathbb{N}$ and $n \in \mathbb{Z}_{+}$such that $m+n>1$. Choose $f_{1} \in$ $\mathcal{H}_{V}(1, \ell), f_{2} \in \mathcal{H}_{V}(1, m), f_{3} \in \mathcal{H}_{V}(1, n)$ and integers $1 \leq i \leq m+n-1$ and $1 \leq j \leq n$. One of the following 3 holds:

(1) $i<j$ and as a formal series in $t_{1}$ and $t_{2}$

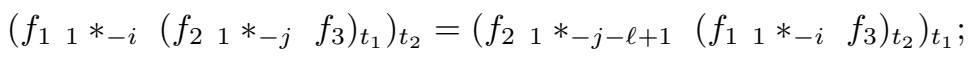

(2) $j \leq i<j+m$ and as a formal series in $t_{1}$ and $t_{2}$

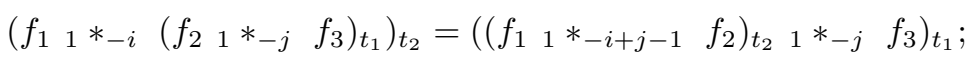

(3) $i \geq j+m$ and as a formal series in $t_{1}$ and $t_{2}$

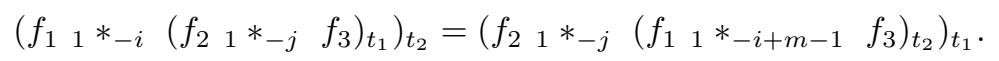

Since this proposition shows equality as formal power series, it also implies absolute convergence of both sides and equality given the absolute convergence of either side.

Moving from associativity to permutations, the symmetric group on $n$ letters acts naturally on $\overline{V^{\otimes n}}$ from the left, i.e. it is determined by

$$
\sigma\left(v_{1} \otimes \ldots \otimes v_{n}\right)=v_{\sigma^{-1}(1)} \otimes v_{\sigma^{-1}(n)}
$$

for all $\sigma \in S_{n}$ and $v_{1}, \ldots, v_{n} \in V$. This induces a left action on $\mathcal{H}_{V}(1, n)$ given by

$$
\sigma(f)(v)=\sigma(f(v)) .
$$


Transpositions play a fundamental role in the actions of the symmetric group so we will make heavy use of the transposition map

$$
\begin{aligned}
T: V \otimes V & \rightarrow V \otimes V \\
v \otimes w & \mapsto w \otimes v .
\end{aligned}
$$

Hence, from the definition of $t$-contraction and the action of the symmetric group we see the following.

Proposition 3.2. Let $f_{1}, f_{2} \in \mathcal{H}_{V}(1,2)$. Then $f_{1}{ }_{1} \infty_{-1} f_{2}$ exists if and only if $\left(I d_{V} \otimes T\right)\left(T \otimes I d_{V}\right)\left(f_{1}{ }_{1} \infty_{-2}\left(T f_{2}\right)\right)$ also exists. If this is the case,

$$
f_{1} *_{-1} \quad f_{2}=\left(I d_{V} \otimes T\right)\left(T \otimes I d_{V}\right)\left(f_{1}{ }_{1} *_{-2} \quad\left(T f_{2}\right)\right) .
$$

Proposition 3.2 implies that all symmetric groups act functorially with respect to contraction since every symmetric group is generated by transpositions.

Remark 3.3. In his book [H2], Huang examines a similar $t$-contraction and contraction on the set of linear maps $\mathcal{H}_{V}(m, 1)$ for $m \in \mathbb{Z}$. The vector spaces $\mathcal{H}_{V}(m, 1)$ and $\mathcal{H}_{V}(1, m)$ are naturally isomorphic, and it can even be shown that the $t$ contraction and contraction in $\mathcal{H}_{V}(m, 1)$ correspond to the $t$-contraction and contraction in $\mathcal{H}_{V}(1, m)$ under this isomorphism. Geometric vertex operator algebras arise from considering maps $\nu_{m}: K(m) \rightarrow H(m, 1)$ such that the diagram

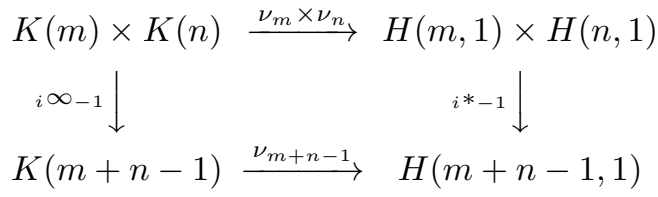

commutes (up to scalars, when the map ${ }_{i} \infty_{-1}$ is defined). Among other conditions for GVOAs, the grading and meromorphicity axioms place additional requirements on the image of $\left\langle v^{\prime}, \nu_{m}(Q) v\right\rangle$ that depend on the choice of elements $Q \in K(m)$ and $v \in V^{\otimes m}$, but not on $v^{\prime} \in V^{\prime}$. In some sense we could use the isomorphisms $K(m) \cong K^{*}(m)$ (which are not canonical - recall Remark 2.17) and $H(m, 1) \cong$ $H(1, m)$ to achieve the diagram

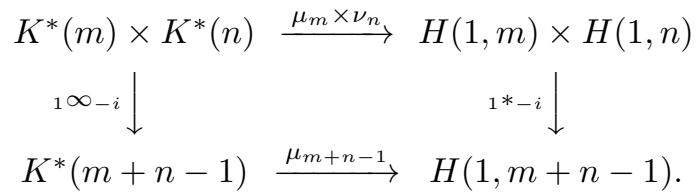

This diagram underlies geometric vertex operator coalgebras. However, in order to maintain consistency with the current worldsheet model, GVOCs must still place additional requirements on the image of $\left\langle v^{\prime}, \mu_{m}(Q) v\right\rangle$ that depend on the choice of elements $Q \in K^{*}(m)$ and $v \in V$, but not on $v^{\prime} \in\left(V^{\otimes m}\right)^{\prime}$. These requirements would be dualized under the above isomorphisms and hence would be incorrect. The approach we provide in Section $\mathbf{5}$ yields a constructive isomorphism with a constructive inverse (that is natural with respect to the formal definition of $K^{*}$ ). The above approach is both nonconstructive and not canonical so that, even if the discrepancies with the grading and meromorphicity axioms were resolved, our approach produces a more useful outcome. 
3.3. More on sewing identities in representations of the Virasoro algebra. Now that we have developed another object on which the Virasoro algebra may act, it is important to highlight the independence of the choice of the $\Theta$ sequences from the representation in which they are selected. Without specifying the specific action of the Virasoro algebra on a complex vector space $V$, we can claim the sewing identities we discussed in Section $2.8(\sqrt{2.26})$ and (2.28) $)$. They correspond to Propositions 4.3.9 and 4.3.10 from [H2] with minor modifications.

Proposition 3.4. Let $\Theta^{(2)}=\Theta^{(2)}\left(\mathcal{A}, \alpha_{0}^{(1)}, \zeta\right), \Theta_{0}^{(2)}=\Theta_{0}^{(2)}\left(\mathcal{A}, \alpha_{0}^{(1)}, \zeta\right)$ be chosen as in Proposition [2.20 to depend on $\mathcal{A}, \alpha_{0}^{(1)}$, and $\zeta$.

Then in the algebra $($ End $V)[[t]]\left[y, y^{-1}\right]\left[\alpha_{0}^{(1)}, \alpha_{0}^{(1)}\right]\left[\left[\mathcal{A}^{(1)}\right]\right]$ we have

$$
\begin{aligned}
e^{\sum_{k=-1}^{\infty}\left(\sum_{j \in \mathbb{Z}_{+}}\left(\alpha_{0}^{(1)}\right)^{-j} \mathcal{A}_{j}^{(1)}\left(\begin{array}{c}
j+1 \\
k+1
\end{array}\right) \zeta^{-j-k}\right) L(-k)} & \\
& =e^{-\Theta_{0}^{(2)} L(0)} e^{-\sum_{j \in \mathbb{Z}_{+}} \Theta_{j}^{(2)} L(-j)} e^{\left(-\zeta+\hat{f}_{2}(\zeta)\right) L(1)} .
\end{aligned}
$$

Proposition 3.5. Similarly, let $\Theta^{(1)}=\Theta^{(1)}(\mathcal{B}, z), \Theta_{0}^{(1)}=\Theta_{0}^{(1)}(\mathcal{B}, z)$ be as in Equation [2.28. Then in the algebra $($ End $V)[[t]]\left[y, y^{-1}\right]\left[\left[\mathcal{B}^{(-1)}\right]\right]$ we have

$$
\begin{aligned}
& e^{-\sum_{k=-1}^{\infty}\left(\sum_{j \in \mathbb{Z}_{+}}\left(\begin{array}{c}
j+1 \\
k+1
\end{array}\right) \mathcal{B}_{j}^{(-1)} z^{j-k}\right) L(-k)} \\
& =e^{-\Theta_{0}^{(1)} L(0)} e^{-\sum_{j \in \mathbb{Z}_{+}} \Theta_{j}^{(1)} L(-j)} e^{\left(-z+\hat{f}_{1}^{-1}(z)\right) L(1)} .
\end{aligned}
$$

\section{The Notion of VerTex operator COALGEbra AND its GEOMETRIC} INTERPRETATION

We now have the background to define the notion of (algebraic) vertex operator coalgebra axiomatically as well as the notion of geometric vertex operator coalgebra.

4.1. The notion of geometric vertex operator coalgebra. We begin by defining the primary geometric motivation for vertex operator coalgebras. The definition of a geometric vertex operator coalgebra uses the same kind of conformal structure that underlies the definition of a geometric vertex operator algebra first given in [H1] and [H2 (in order that they might eventually be combined). One may think of a geometric vertex operator algebra as a meromorphic morphism (or algebra) associated to a $\mathbb{C}$-extension of the partial operad $K$. (See Section 6 of HL.) Similarly, a geometric vertex operator coalgebra may be interpreted as a meromorphic morphism (or coalgebra) of a $\mathbb{C}$-extension of the partial operad $K^{*}$ to the partial pseudo-operad $\left\{\mathcal{H}_{V}(1, n)\right\}_{n \in \mathbb{N}}$. We will not focus on this interpretation here.

Definition 4.1. A geometric vertex operator coalgebra (over $\mathbb{C}$ ) of rank $d \in \mathbb{C}$ is a $\mathbb{Z}$-graded vector space over $\mathbb{C}$

$$
V=\coprod_{k \in \mathbb{Z}} V_{(k)}
$$

such that $\operatorname{dim} V_{(k)}<\infty$ for $k \in \mathbb{Z}$ and $V_{(k)}=0$ for $k$ sufficiently small, together with a linear map for each $n \in \mathbb{N}$

$$
\mu_{n}: K^{*}(n) \mapsto \mathcal{H}_{V}(1, n)
$$


satisfying the following axioms:

1. Grading: Let $k \in \mathbb{Z}, v \in V_{(k)}, v^{\prime} \in V^{\prime}$, and $a \in \mathbb{C}^{\times}$. Then

$$
\left\langle v^{\prime}, \mu_{1}(\mathbf{0},(a, \mathbf{0})) v\right\rangle=a^{-k}\left\langle v^{\prime}, v\right\rangle .
$$

2. Meromorphicity: For any $n \in \mathbb{N}, v^{\prime} \in\left(V^{\otimes n}\right)^{\prime}$, and $v \in V$ the function

$$
Q \mapsto\left\langle v^{\prime}, \mu_{n}(Q) v\right\rangle
$$

from $K^{*}(n)$ to $\mathbb{C}$ is a meromorphic function on $K^{*}(n)$. Further, for any $v \in V$ there exists $N(v) \in \mathbb{Z}_{+}$such that for all $v^{\prime} \in\left(V^{\otimes n}\right)^{\prime}$ the degree of $z_{-i}$ in $\left\langle v^{\prime}, \mu_{n}(Q) v\right\rangle$ is less than $N(v)$, for $i=1, \ldots, n-1$.

3. Permutation: Let $\sigma \in S_{n}$. Then for any $Q \in K^{*}(n)$

$$
\sigma\left(\mu_{n}(Q)\right)=\mu_{n}(\sigma(Q))
$$

4. Sewing: Given

$$
\begin{gathered}
Q_{1}=\left(z_{-m+1}^{-1}, \ldots, z_{-1}^{-1} ; A^{(-m)},\left(a_{0}^{(-m+1)}, A^{(-m+1)}\right), \ldots,\left(a_{0}^{(-1)}, A^{(-1)}\right),\right. \\
\left.\left(a_{0}^{(1)}, A^{(1)}\right)\right) \in K^{*}(m), \\
Q_{2}=\left(\zeta_{-n+1}^{-1}, \ldots, \zeta_{-1}^{-1} ; B^{(-n)},\left(b_{0}^{(-n+1)}, B^{(-n+1)}\right), \ldots,\left(b_{0}^{(-1)}, B^{(-1)}\right),\right. \\
\left.\left(b_{0}^{(1)}, B^{(1)}\right)\right) \in K^{*}(n),
\end{gathered}
$$

if the $i$-th outgoing puncture $(1 \leq i \leq n)$ of $Q_{2}$ can be sewn to the incoming puncture of $Q_{1}$, then for any $v^{\prime} \in\left(V^{\otimes m+n-1}\right)^{\prime}, v \in V$

$$
\left\langle v^{\prime},\left(\mu_{m}\left(Q_{1}\right)_{1} *_{-i} \quad \mu_{n}\left(Q_{2}\right)_{t}(v)\right\rangle\right.
$$

is absolutely convergent when $t=1$, and

$$
\mu_{m+n-1}\left(Q_{1}{ }_{1} \infty_{-i} Q_{2}\right)=\left(\mu_{n}\left(Q_{1}\right)_{1} *_{-i} \mu_{m}\left(Q_{2}\right) e^{-\Gamma\left(A^{(1)}, B^{(-i)}, a_{0}^{(1)} b_{0}^{(-i)}\right) d}\right.
$$

where $d$ is the rank and $\Gamma\left(A^{(1)}, B^{(-i)}, a_{0}^{(1)} b_{0}^{(-i)}\right)$ is as in 2.24).

We denote the geometric vertex operator coalgebra just defined by $(V, \mu=$ $\left\{\mu_{n}\right\}_{n \in \mathbb{N}}$ ) or, when there is no ambiguity, simply $V$.

Remark 4.2. The second half of the meromorphicity axiom may be interpreted as saying that $\left\langle v^{\prime}, \mu_{n}(Q) v\right\rangle$, when viewed as a function in $z_{-1}^{-1}, \ldots, z_{-n+1}^{-1}$, has poles at $z_{-i}^{-1}=0$ (for $\left.i=1, \ldots, n-1\right)$ and $z_{-i}^{-1}=z_{-j}^{-1}$ (for $i \neq j$ ) such that the order of each of these poles is bounded by $N(v)$ independent of $v^{\prime} \in\left(V^{\otimes n}\right)^{\prime}$. This fact comes directly from the meromorphicity axiom for poles at $z_{i}^{-1}=0$ but for poles at $z_{i}^{-1}=$ $z_{j}^{-1}$ this observation involves the sewing axiom. Incidently, the meromorphicity axiom of a geometric vertex operator algebra includes a small bit of redundancy in that the order of the poles at $z_{i}=z_{j}($ for $i \neq j)$ is bounded as a result of the bounding of poles at $z_{i}=0$ (for $\left.i=1, \ldots, n-1\right)$ and the sewing axiom. 
4.2. The notion of vertex operator coalgebra. The following description of a vertex operator coalgebra is the central definition of this paper. It is in a sense the culmination of our effort to algebraically understand the structure induced on vector spaces by moduli spaces of spheres with outgoing tubes. In another sense, however, it is a starting point for algebraic study. A few algebraic properties will be described in this section but these barely scratch the surface in reference to questions about the structure of vertex operator coalgebras and representations over them. See Hub2] and Hub3] for additional exploration into the algebraic properties of VOCs.

Definition 4.3. $A$ vertex operator coalgebra (over $\mathbb{C}$ ) of rank $d \in \mathbb{C}$ is a $\mathbb{Z}$-graded vector space over $\mathbb{C}$

$$
V=\coprod_{k \in \mathbb{Z}} V_{(k)}
$$

such that $\operatorname{dim} V_{(k)}<\infty$ for $k \in \mathbb{Z}$ and $V_{(k)}=0$ for $k$ sufficiently small, together with linear maps

$$
\begin{gathered}
X(x): V \mapsto(V \otimes V)\left[\left[x, x^{-1}\right]\right] \\
v \mapsto X(x) v=\sum_{k \in \mathbb{Z}} \Delta_{k}(v) x^{-k-1}, \\
c: V \mapsto \mathbb{C} \\
\rho: V \mapsto \mathbb{C}
\end{gathered}
$$

called the coproduct, the covacuum map and the co-Virasoro map, respectively, satisfying the following 7 axioms:

1. Left Counit: For all $v \in V$

$$
\left(c \otimes I d_{V}\right) X(x) v=v
$$

2. Cocreation: For all $v \in V$

$$
\begin{gathered}
\left(I d_{V} \otimes c\right) X(x) v \in V[[x]] \text { and } \\
\lim _{x \rightarrow 0}\left(I d_{V} \otimes c\right) X(x) v=v .
\end{gathered}
$$

3. Truncation: Given $v \in V$, then $\Delta_{k}(v)=0$ for $k$ sufficiently small.

4. Jacobi Identity:

$$
\begin{array}{r}
x_{0}^{-1} \delta\left(\frac{x_{1}-x_{2}}{x_{0}}\right)\left(I d_{V} \otimes X\left(x_{2}\right)\right) \Lambda\left(x_{1}\right)-x_{0}^{-1} \delta\left(\frac{x_{2}-x_{1}}{-x_{0}}\right)\left(T \otimes I d_{V}\right) \\
\left(I d_{V} \otimes X\left(x_{1}\right)\right) \Lambda\left(x_{2}\right)=x_{2}^{-1} \delta\left(\frac{x_{1}-x_{0}}{x_{2}}\right)\left(X\left(x_{0}\right) \otimes I d_{V}\right) \Lambda\left(x_{2}\right) .
\end{array}
$$

5. Virasoro Algebra: The Virasoro algebra bracket, 


$$
[L(j), L(k)]=(j-k) L(j+k)+\frac{1}{12}\left(j^{3}-j\right) \delta_{j,-k} d,
$$

holds for $j, k \in \mathbb{Z}$, where

$$
\left(\rho \otimes I d_{V}\right) \Lambda(x)=\sum_{k \in \mathbb{Z}} L(k) x^{k-2} .
$$

6. Grading: For each $k \in \mathbb{Z}$ and $v \in V_{(k)}$

$$
L(0) v=k v .
$$

7. L(1)-Derivative:

$$
\frac{d}{d x} X(x)=\left(L(1) \otimes I d_{V}\right) X(x) .
$$

We denote this vertex operator coalgebra by $(V, \lambda, c, \rho)$, or sometimes just $V$.

Note that $x, x_{0}, x_{1}$ and $x_{2}$ are formal commuting variables and $X$ is linear so that, for example, $\left(I d_{V} \otimes X\left(x_{1}\right)\right)$ acting on the coefficients of $X\left(x_{2}\right) v \in(V \otimes V)\left[\left[x_{2}, x_{2}^{-1}\right]\right]$ is well defined. Notice also, that when each expression is applied to any element of $V$, the coefficient of each monomial in the formal variables is a finite sum.

Remark 4.4. Note that while the definition of vertex operator coalgebra is stated in such a way as to maximize its resemblance to that of a vertex operator algebra (as presented in [FLM], [FHL, and [LL) there are several important differences. First, whereas the creation and truncation axioms of VOAs both bound the power of the formal variable from below, in VOCs cocreation allows only non-negative powers of $x$ while truncation allows only finitely many positive powers of $x$ but infinitely many negative powers. Among other implications, this means that cocreation actually generates polynomials in $x$. Second, note that the representation of the Virasoro algebra has been inverted, then shifted by $x^{-4}$. Finally, while the Jacobi identity description highlights similarities between VOAs and VOCs, examining the corresponding "weak commutativity" properties reveals substantial differences (cf. [FHL] and $\mathrm{Hub3}$ ).

4.3. Properties of VOCs. There are a number of interesting consequences of the above axioms, most quite analogous to VOA properties. Properties are listed here without proof but the reader is encouraged to see Hub1 or Hub3 for more adequate justifications.

$$
\left(e^{x_{0} L(1)} \otimes I d_{V}\right) X(x)=\Lambda\left(x+x_{0}\right) .
$$

The L(1)-commutation formula:

$$
\Lambda\left(x_{2}\right) L(1)=\left(I d_{V} \otimes L(1)\right) X\left(x_{2}\right)+\left(L(1) \otimes I d_{V}\right) X\left(x_{2}\right)
$$

The $L(0)$-commutation formula:

$$
X\left(x_{2}\right) L(0)=\left(I d_{V} \otimes L(0)\right) X\left(x_{2}\right)+\left(L(0) \otimes I d_{V}\right) X\left(x_{2}\right)+x_{2}\left(L(1) \otimes I d_{V}\right) X\left(x_{2}\right)
$$


The $L(-1)$-commutation formula:

$\Lambda\left(x_{2}\right) L(-1)=\left(I d_{V} \otimes L(-1)\right) X\left(x_{2}\right)+\left(\left(x_{2}^{2} L(1)+2 x_{2} L(0)+L(-1)\right) \otimes I d_{V}\right) X\left(x_{2}\right)$

$$
\begin{aligned}
& \Lambda\left(x_{2}\right) \sum_{k \in \mathbb{Z}} L(k) x_{1}^{k-2}-\left(I d_{V} \otimes \sum_{k \in \mathbb{Z}} L(k) x_{1}^{k-2}\right) \Lambda\left(x_{2}\right) \\
& =\operatorname{Res}_{x_{0}} x_{2}^{-1} \delta\left(\frac{x_{1}-x_{0}}{x_{2}}\right)\left(\sum_{k \in \mathbb{Z}} L(k) x_{0}^{k-2} \otimes I d_{V}\right) X\left(x_{2}\right) \\
& \left(e^{x_{0} L(1)} \otimes I d_{V}\right) X(x)=\left(I d_{V} \otimes e^{-x_{0} L(1)}\right) X(x) e^{x_{0} L(1)} \\
& e^{x_{0} L(1)}=\left(I d_{V} \otimes c\right) \Lambda\left(x_{0}\right)
\end{aligned}
$$

Anti-symmetry:

$$
\begin{gathered}
T \Lambda(x)=\Lambda(-x) e^{x L(1)} \\
c L(j)=0 \in \operatorname{Hom}(V, \mathbb{C}) \text { for } j \leq 1 \\
c L(2)=\rho \\
\rho L(0)=2 \rho
\end{gathered}
$$

Several fundamental facts about VOCs necessitate an investigation of the weights of vectors and operators. Among other things, weights help us to understand the elements $\Delta_{n}(v) \in V \otimes V$. When $v \in V_{(k)}$, we say that $v$ is homogeneous of weight $k$, and the grading axiom says $L(0) v=($ wt $v) v$. Given an element $w \in V \otimes V$, if $\left(\left(L(0) \otimes I d_{V}\right)+\left(I d_{V} \otimes L(0)\right)\right) w=a w$ for some $a \in \mathbb{C}$, then we say that the weight of $w$ is $a$. This agrees with the grading on $V \otimes V$ since for $v \in V_{(k)}$ and $w \in V_{(\ell)}$, we have $v \otimes w \in(V \otimes V)_{(k+\ell)}$ and wt $(v \otimes w)=k+\ell$. With this motivation, for $v \in V_{(k)}$ and $w \in V_{(\ell)}$, we define $x^{I d_{V} \otimes L(0)}(v \otimes w)=x^{\ell}(v \otimes w)$ and $x^{L(0) \otimes I d_{V}}(v \otimes w)=x^{k}(v \otimes w)$. For homogeneous vectors, we see that

$$
\text { wt } \Delta_{j}(v)=\text { wt }(v)+j+1 \text {. }
$$

Considering $v \in V_{(k)}$, it is now clear via (4.19) that for each $\Delta_{j}(v)$,

$$
\Delta_{j}(v)=\sum_{\ell=1}^{r_{j}} u_{\ell} \otimes w_{\ell}
$$

where each $u_{\ell} \in V_{\left(i_{j}\right)}$, each $w_{\ell} \in V_{\left(\mathrm{wt} \Delta_{j}(v)-i_{j}\right)}, r_{j} \in \mathbb{Z}_{+}$, and each $i_{j} \in \mathbb{Z}$. We also have:

$$
x^{-I d_{V} \otimes L(0)} \Lambda(x) x_{0}^{L(0)}=x_{0}^{L(0) \otimes I d_{V}} \Lambda\left(x_{0} x\right)
$$




$$
\begin{gathered}
\left(I d_{V} \otimes a^{-L(0)}\right) X(x) a^{L(0)}=\left(a^{L(0)} \otimes I d_{V}\right) X(a x) \text { for } a \in \mathbb{C}^{\times} \\
a^{L(0)} e^{b L(k)}=e^{a^{-k} b L(k)} a^{L(0)} \text { for } a \in \mathbb{C}^{\times}, b \in \mathbb{C}, k \in \mathbb{Z} .
\end{gathered}
$$

Given $v \in V_{(k)}$, the fact that

$$
L(0) L(j) v=(k-j) L(j) v
$$

for any $j, k \in \mathbb{Z}$, tells us that each operator $L(j)$ raises the weight of homogeneous vectors by $-j$ and is said to have weight $-j$, as is the case with VOAs.

The Jacobi identity may be replaced in the definition of VOC with properties called right and left rationality, commutativity, and associativity as the following two propositions show. Let $\iota_{1} 2$ map rational functions in $x_{1}$ and $x_{2}$ to their expansion in only finitely many negative powers of $x_{2}$. Define $\iota_{2} 1$ and $\iota_{2} 0$ similarly (cf. Section 3.1 of $[\mathrm{FHL}$ ).

Proposition 4.5. Let $(V, X, c, \rho)$ be a VOC. For any $v^{\prime} \in\left(V^{\otimes 3}\right)^{\prime}$ and $v \in V$ we have the following properties.

(i) Right rationality:

$$
\left\langle v^{\prime},\left(I d_{V} \otimes X\left(x_{2}\right)\right) X\left(x_{1}\right) v\right\rangle=\iota_{1} \frac{g\left(x_{1}, x_{2}\right)}{x_{1}^{r} x_{2}^{s}\left(x_{1}-x_{2}\right)^{t}}
$$

for some $g\left(x_{1}, x_{2}\right) \in \mathbb{C}\left[x_{1}, x_{2}\right]$ and $r, s, t \in \mathbb{Z}$, where $g\left(x_{1}, x_{2}\right)$ is unique up to choice of $r, s$ and $t$.

(ii) Left rationality:

$$
\left\langle v^{\prime},\left(X\left(x_{0}\right) \otimes I d_{V}\right) X\left(x_{2}\right) v\right\rangle=\iota_{2} 0 \frac{h\left(x_{0}, x_{2}\right)}{x_{0}^{r} x_{2}^{s}\left(x_{0}+x_{2}\right)^{t}}
$$

for some $h\left(x_{0}, x_{2}\right) \in \mathbb{C}\left[x_{0}, x_{2}\right]$ and $r, s, t \in \mathbb{Z}$, again with $h\left(x_{0}, x_{2}\right)$ unique up to choice of $r, s$ and $t$.

(iii) Commutativity:

$$
\iota_{12}^{-1}\left\langle v^{\prime},\left(I d_{V} \otimes X\left(x_{2}\right)\right) X\left(x_{1}\right) v\right\rangle=\iota_{2}^{-1}\left\langle v^{\prime},\left(T \otimes I d_{V}\right)\left(I d_{V} \otimes X\left(x_{1}\right)\right) X\left(x_{2}\right) v\right\rangle .
$$

(iv) Associativity:

$$
\iota_{12}^{-1}\left\langle v^{\prime},\left(I d_{V} \otimes X\left(x_{2}\right)\right) X\left(x_{1}\right) v\right\rangle=\left.\left(\iota_{20}^{-1}\left\langle v^{\prime},\left(X\left(x_{0}\right) \otimes I d_{V}\right) X\left(x_{2}\right) v\right\rangle\right)\right|_{x_{0}=x_{1}-x_{2}} .
$$

Proposition 4.6. In the presence of the other VOC axioms, right and left rationality, commutativity, and associativity imply the Jacobi identity.

For the proofs of Propositions 4.5 and 4.6 see Hub1 or Hub3. 


\section{An isomorphism Between the CATEgories of GVOCs ANd VOCs}

We defined geometric vertex operator coalgebras to motivate the definition of vertex operator coalgebras. We are now in a position to prove that these definitions actually define isomorphic categories. The category of GVOCs of rank $d$ has the set of all GVOCs of rank $d$ as its objects and its morphisms are those linear maps between GVOCs that are $\mu$ invariant. The category of VOCs of rank $d$ has all VOCs of rank $d$ as objects and for morphisms, linear maps between VOCs which are coproduct invariant as well as preserving the covacuum and co-Virasoro maps.

We will define a functor from GVOCs to VOCs and then a functor from VOCs to GVOCs. Finally, we will show that these two functors are inverses to each other, thus giving an isomorphism.

5.1. A map from GVOCs to VOCs. In this section we construct a map from the category of geometric vertex operator coalgebras to the category of vertex operator coalgebras. In Section 5.3 we will prove that, in fact, this map is an isomorphism.

Let $(V, \mu)$ be a geometric vertex operator coalgebra. We define a linear map $c^{\mu}: V \rightarrow \mathbb{C}$ by

$$
c^{\mu}=\mu_{0}((1, \mathbf{0}))
$$

a linear map $\rho^{\mu}: V \rightarrow \mathbb{C}$ by

$$
\rho^{\mu}=-\left.\frac{d}{d \varepsilon} \mu_{0}(1,(0, \varepsilon, 0,0, \ldots))\right|_{\varepsilon=0},
$$

and a linear map $X^{\mu}: V \mapsto(V \otimes V)\left[\left[x, x^{-1}\right]\right]$ by

$$
\operatorname{Res}_{x} x^{n}\left\langle v^{\prime}, X^{\mu}(x) v\right\rangle=\operatorname{Res}_{z} z^{n}\left\langle v^{\prime}, \mu\left(\left(z^{-1} ; \mathbf{0},(1, \mathbf{0}),(1, \mathbf{0})\right)\right) v\right\rangle,
$$

for $v^{\prime} \in(V \otimes V)^{\prime}, v \in V$ where $\operatorname{Res}_{x}$ means taking the coefficient of the $x^{-1}$ term in the given series and $\operatorname{Res}_{z}$ means taking the residue of the function at the singularity $z=0$.

Proposition 5.1. If the rank of $(V, \mu)$ is $d$, then the quadruple $\left(V, X^{\mu}, c^{\mu}, \rho^{\mu}\right)$ is a vertex operator coalgebra of rank $d$.

Proof. We use the GVOC axioms to prove the VOC axioms with respect to $\left(V, X^{\mu}, c^{\mu}, \rho^{\mu}\right)$.

1. Left Counit:

$$
\begin{aligned}
\left.\left\langle v^{\prime},\left(c^{\mu} \otimes I d_{V}\right) X^{\mu}(x)(v)\right\rangle\right|_{x=z} & =\left.\left\langle v^{\prime},\left(\mu_{0}((1, \mathbf{0})) \otimes I d_{V}\right) X^{\mu}(x) v\right\rangle\right|_{x=z} \\
& =\left\langle v^{\prime}, \mu_{0}((1, \mathbf{0}))_{1} *_{-1} \quad \mu_{2}\left(\left(z^{-1} ; \mathbf{0},(1, \mathbf{0}),(1, \mathbf{0})\right)\right) v\right\rangle \\
& =\left\langle v^{\prime}, \mu_{1}\left((1, \mathbf{0})_{1} \infty_{-1}\left(z^{-1} ; \mathbf{0},(1, \mathbf{0}),(1, \mathbf{0})\right)\right) v\right\rangle \\
& =\left\langle v^{\prime}, \mu_{1}(\mathbf{0},(1, \mathbf{0})) v\right\rangle \\
& =\left\langle v^{\prime}, v\right\rangle .
\end{aligned}
$$

(The second equality uses projection maps to show convergence and the last step uses a trivial application of the grading axiom.)

2. Cocreation: 
KEITH HUBBARD

$$
\begin{aligned}
\left.\left\langle v^{\prime},\left(I d_{V} \otimes c^{\mu}\right) X^{\mu}(x)(v)\right\rangle\right|_{x=z} & =\left.\left\langle v^{\prime},\left(I d_{V} \otimes \mu_{0}((1, \mathbf{0}))\right) \Lambda^{\mu}(x) v\right\rangle\right|_{x=z} \\
& =\left\langle v^{\prime}, \mu_{0}((1, \mathbf{0}))_{1} *_{-2} \quad \mu_{2}\left(\left(z^{-1} ; \mathbf{0},(1, \mathbf{0}),(1, \mathbf{0})\right)\right) v\right\rangle \\
& =\left\langle v^{\prime}, \mu_{1}\left((1, \mathbf{0})_{1} \infty_{-2}\left(z^{-1} ; \mathbf{0},(1, \mathbf{0}),(1, \mathbf{0})\right)\right) v\right\rangle \\
& =\left\langle v^{\prime}, \mu_{1}(\mathbf{0},(1,(-z, 0,0, \ldots))) v\right\rangle .
\end{aligned}
$$

By the meromorphicity axiom, $\left\langle v^{\prime}, \mu_{1}(\cdot) v\right\rangle$ is a meromorphic function on $K^{*}(1)$, so $\left\langle v^{\prime}, \mu_{1}(\mathbf{0},(1,(-z, 0,0, \ldots))) v\right\rangle$ is a polynomial in $z$. Thus for all $v \in V, v^{\prime} \in V^{\prime}$, $\left\langle v^{\prime},\left(I d_{V} \otimes c^{\mu}\right) X^{\mu}(x)(v)\right\rangle \in V[x]$. We can also see that

$$
\begin{aligned}
\lim _{z \rightarrow 0}\left\langle v^{\prime}, \mu_{1}(\mathbf{0},(1,(-z, 0,0, \ldots))) v\right\rangle & =\left\langle v^{\prime}, \mu_{1}(\mathbf{0},(1, \mathbf{0})) v\right\rangle \\
& =\left\langle v^{\prime}, v\right\rangle .
\end{aligned}
$$

3. Truncation:

By the meromorphicity axiom, for any $v \in V$ there exists $N(v) \in \mathbb{Z}_{+}$such that for all $v^{\prime} \in(V \otimes V)^{\prime}$, the power of $z$ in $\left\langle v^{\prime}, \mu_{2}\left(\left(z^{-1} ; \mathbf{0},(1, \mathbf{0}),(1, \mathbf{0})\right)\right) v\right\rangle$ is less than $N(v)$. But

$$
\left\langle v^{\prime}, \mu_{2}\left(\left(z^{-1} ; \mathbf{0},(1, \mathbf{0}),(1, \mathbf{0})\right)\right) v\right\rangle=\left.\left\langle v^{\prime}, X^{\mu}(x) v\right\rangle\right|_{x=z},
$$

so the number of positive powers of $\mathrm{x}$ in $X^{\mu}(x) v$ must be less than $N(v)$ as well. 4. Jacobi Identity:

The idea behind proving the Jacobi identity is to prove right and left rationality as well as commutativity and associativity. Appealing to Proposition 4.6 this is equivalent to the Jacobi identity.

We will start by obtaining right rationality and commutativity.

$$
\begin{aligned}
\left\langle v^{\prime},\left(I d_{V}\right.\right. & \left.\left.\otimes X^{\mu}\left(x_{2}\right)\right) X^{\mu}\left(x_{1}\right) v\right\rangle\left.\right|_{x_{i}=z_{-i}} \\
& =\left\langle v^{\prime},\left(I d_{V} \otimes \mu_{2}\left(\left(z_{-2}^{-1} ; \mathbf{0},(1, \mathbf{0}),(1, \mathbf{0})\right)\right)\right) \mu_{2}\left(\left(z_{-1}^{-1} ; \mathbf{0},(1, \mathbf{0}),(1, \mathbf{0})\right)\right) v\right\rangle \\
& =\left\langle v^{\prime}, \mu_{3}\left(\left(z_{-2}^{-1} ; \mathbf{0},(1, \mathbf{0}),(1, \mathbf{0})\right)_{1} \infty_{-2}\left(z_{-1}^{-1} ; \mathbf{0},(1, \mathbf{0}),(1, \mathbf{0})\right)\right) v\right\rangle \\
& =\left\langle v^{\prime}, \mu_{3}\left(\left(z_{-1}^{-1}, z_{-2}^{-1} ; \mathbf{0},(1, \mathbf{0}),(1, \mathbf{0}),(1, \mathbf{0})\right)\right) v\right\rangle
\end{aligned}
$$

for any $z_{-1}, z_{-2} \in \mathbb{C}^{\times}$for which this sewing is well defined, i.e., for $\left|z_{-1}\right|>\left|z_{-2}\right|$. Similarly,

$$
\left.\left\langle v^{\prime},\left(I d_{V} \otimes \Lambda^{\mu}\left(x_{1}\right)\right) \Lambda^{\mu}\left(x_{2}\right) v\right\rangle\right|_{x_{i}=z_{-i}}=\left\langle v^{\prime}, \mu_{3}\left(\left(z_{-2}^{-1}, z_{-1}^{-1} ; \mathbf{0},(1, \mathbf{0}),(1, \mathbf{0}),(1, \mathbf{0})\right)\right) v\right\rangle
$$

for $\left|z_{-2}\right|>\left|z_{-1}\right|$. By the meromorphicity axiom,

$$
\left\langle v^{\prime}, \mu_{3}\left(\left(z_{-1}^{-1}, z_{-2}^{-1} ; \mathbf{0},(1, \mathbf{0}),(1, \mathbf{0}),(1, \mathbf{0})\right)\right) v\right\rangle=\frac{g\left(z_{-1}, z_{-2}\right)}{z_{-1}^{r} z_{-2}^{s}\left(z_{-1}-z_{-2}\right)^{t}}
$$

where $g\left(z_{-1}, z_{-2}\right) \in \mathbb{C}\left[z_{-1}, z_{-2}\right]$ and $r, s, t \in \mathbb{Z}$. Thus, since

$$
\left.\left\langle v^{\prime},\left(I d_{V} \otimes X^{\mu}\left(x_{2}\right)\right) X^{\mu}\left(x_{1}\right) v\right\rangle\right|_{x_{i}=z_{-i}}=\frac{g\left(z_{-1}, z_{-2}\right)}{z_{-1}^{r} z_{-2}^{s}\left(z_{-1}-z_{-2}\right)^{t}}
$$


and $\left\langle v^{\prime},\left(I d_{V} \otimes X^{\mu}\left(x_{2}\right)\right) X^{\mu}\left(x_{1}\right) v\right\rangle$ has only finitely many positive powers of $x_{1}$ and hence only finitely many negative powers of $x_{2}$ by evaluating weights on homogeneous vectors,

$$
\left\langle v^{\prime},\left(I d_{V} \otimes X^{\mu}\left(x_{2}\right)\right) X^{\mu}\left(x_{1}\right) v\right\rangle=\iota_{1} 2 \frac{g\left(x_{1}, x_{2}\right)}{x_{1}^{r} x_{2}^{s}\left(x_{1}-x_{2}\right)^{t}},
$$

i.e., $V$ satisfies right rationality. By the permutation axiom,

$$
\begin{aligned}
\left\langle v^{\prime}, \mu_{3}\left(\left(z_{-1}^{-1}, z_{-2}^{-1} ;(1, \mathbf{0}), \mathbf{0},(1, \mathbf{0}),(1, \mathbf{0})\right)\right) v\right\rangle & \\
= & \left\langle v^{\prime},\left(T \otimes I d_{V}\right) \mu_{3}\left(\left(z_{-2}^{-1}, z_{-1}^{-1} ; \mathbf{0},(1, \mathbf{0}),(1, \mathbf{0})\right)\right) v\right\rangle .
\end{aligned}
$$

Thus

$$
\begin{aligned}
\iota_{12}^{-1}\left\langle v^{\prime},\left(I d_{V} \otimes X^{\mu}\left(x_{2}\right)\right) X^{\mu}\left(x_{1}\right) v\right\rangle & =\frac{g\left(x_{1}, x_{2}\right)}{x_{1}^{r} x_{2}^{s}\left(x_{1}-x_{2}\right)^{t}} \\
& =\iota_{2}^{-1}\left\langle v^{\prime},\left(T \otimes I d_{V}\right)\left(I d_{V} \otimes X^{\mu}\left(x_{1}\right)\right) X^{\mu}\left(x_{2}\right) v\right\rangle
\end{aligned}
$$

proving commutativity. For left rationality and associativity, we use the same technique to argue that

$$
\begin{aligned}
\left\langle v^{\prime},\right. & \left.\left(X^{\mu}\left(x_{0}\right) \otimes I d_{V}\right) X^{\mu}\left(x_{2}\right) v\right\rangle\left.\right|_{x_{0}=\left(z_{-1}-z_{-2}\right), x_{2}=z_{-2}} \\
\quad & =\left\langle v^{\prime}, \mu_{2}\left(\left(\left(z_{-1}-z_{-2}\right)^{-1} ; \mathbf{0},(1, \mathbf{0}),(1, \mathbf{0})\right)\right)_{1} *_{-2} \quad \mu_{2}\left(\left(z_{-2}^{-1} ; \mathbf{0},(1, \mathbf{0}),(1, \mathbf{0})\right)\right) v\right\rangle \\
\quad & =\left\langle v^{\prime}, \mu_{3}\left(\left(z_{-1}, z_{-2} ; \mathbf{0},(1, \mathbf{0}),(1, \mathbf{0}),(1, \mathbf{0})\right)\right) v\right\rangle .
\end{aligned}
$$

for $\left|z_{-2}\right|>\left|z_{-1}-z_{-2}\right|$. Recall that the right-hand side of this equation is equal to

$$
\frac{g\left(z_{-1}, z_{-2}\right)}{z_{-1}^{r} z_{-2}^{s}\left(z_{-1}-z_{-2}\right)^{t}}=\frac{h\left(z_{-1}-z_{-2}, z_{-2}\right)}{\left(\left(z_{-1}-z_{-2}\right)+z_{-2}\right)^{r} z_{-2}^{s}\left(z_{-1}-z_{-2}\right)^{t}}
$$

for some $h\left(z_{-1}-z_{-2}, z_{-2}\right) \in \mathbb{C}\left[z_{-1}, z_{-2}\right]$. Thus as above,

$$
\left\langle v^{\prime},\left(I d_{V}\left(X^{\mu}\left(x_{0}\right) \otimes I d_{V}\right) X^{\mu}\left(x_{2}\right) v\right\rangle=\iota_{2} 0 \frac{h\left(x_{0}, x_{2}\right)}{\left(x_{0}+x_{2}\right)^{r} x_{2}^{s} x_{0}^{t}},\right.
$$

that is, left rationality holds. In addition, we see that

$$
\begin{aligned}
\left(\iota_{2}^{-1}\left\langle v^{\prime},\left(I d_{V}\left(X^{\mu}\left(x_{0}\right) \otimes I d_{V}\right) X^{\mu}\left(x_{2}\right) v\right\rangle\right)\right. & \left.\right|_{x_{0}}=x_{1}-x_{2} \\
& =\frac{h\left(x_{-1}-x_{-2}, x_{-2}\right)}{\left(\left(x_{-1}-x_{-2}\right)+x_{-2}\right)^{r} x_{-2}^{s}\left(x_{-1}-x_{-2}\right)^{t}} \\
& =\iota_{12}^{-1}\left\langle v^{\prime},\left(I d_{V} \otimes X^{\mu}\left(x_{2}\right)\right) X^{\mu}\left(x_{1}\right) v\right\rangle,
\end{aligned}
$$

which is, of course, associativity.

5. Virasoro algebra:

In order to examine the Virasoro algebra structure of the $L(k)$ operators, we must first determine what

$$
\left(\rho \otimes I d_{V}\right) \Lambda(x)=\sum_{k \in \mathbb{Z}} L(k) x^{k-2}
$$


KEITH HUBBARD

forces the $L(k)$ operators to be. We will make use of the linear functionals $\mathcal{L}_{I}(z)$ described in Section 2.9 If we choose $v^{\prime} \in V^{\prime}$ and $v \in V$ then apply $\mathcal{L}_{I}(z)$ to $\left\langle v^{\prime}, \mu_{1}(\cdot) v\right\rangle$, there are two different ways to interpret the result. On the one hand,

$$
\begin{aligned}
\mathcal{L}_{I}(z) & \left\langle v^{\prime}, \mu_{1}(\cdot) v\right\rangle \\
& =\left.\left(\frac{d}{d \varepsilon}\left\langle v^{\prime}, \mu_{1}\left(((1,(0,-\varepsilon, 0,0, \ldots)))_{1} \infty_{-1}\left(z^{-1} ; \mathbf{0},(1, \mathbf{0}),(1, \mathbf{0})\right)\right) v\right\rangle\right)\right|_{\varepsilon=0} \\
& \left.=\left(\frac{d}{d \varepsilon}\left\langle v^{\prime}, \mu_{0}(\mathbf{0},(1,(0,-\varepsilon, 0,0, \ldots)))_{1} *_{-1} X^{\mu}(x)\right) v\right\rangle\right)\left.\right|_{\varepsilon=0, x=z} \\
& \left.=\left.\left\langle v^{\prime}, \frac{-d}{d \varepsilon} \mu_{0}(\mathbf{0},(1,(0, \varepsilon, 0,0, \ldots))) v\right\rangle\right|_{\varepsilon=0} 1_{-1}\left\langle v^{\prime}, X^{\mu}(x)\right) v\right\rangle\left.\right|_{x=z} \\
& =\left.\left\langle v^{\prime},\left(\rho^{\mu} \otimes I d_{V}\right) X^{\mu}(x) v\right\rangle\right|_{x=z}
\end{aligned}
$$

On the other hand, viewing $\mathcal{L}_{I}(z)$ as an element of the tangent space of $K^{*}(1)$ at $(\mathbf{0},(1, \mathbf{0}))$, Lemma 2.24 implies

$$
\begin{aligned}
\mathcal{L}_{I}(z) & \left\langle v^{\prime}, \mu_{1}(\cdot) v\right\rangle \\
= & \left.\sum_{k \in \mathbb{Z}_{+}} z^{-k-2} \frac{-\partial}{\partial A_{k}^{(-1)}}\left\langle v^{\prime}, \mu_{1}\left(\left(a_{0}^{(1)}, A^{(1)}\right), A^{(-1)}\right) v\right\rangle\right|_{A^{(-1)}, A^{(1)}=\mathbf{0}, a_{0}^{(1)}=1} \\
& +\left.z^{-2} \frac{-\partial}{\partial a_{0}^{(1)}}\left\langle v^{\prime}, \mu_{1}\left(\left(a_{0}^{(1)}, A^{(1)}\right), A^{(-1)}\right) v\right\rangle\right|_{A^{(-1)}, A^{(1)}=\mathbf{0}, a_{0}^{(1)}=1} \\
& +\left.\sum_{k \in \mathbb{Z}_{+}} z^{k-2} \frac{-\partial}{\partial A_{k}^{(1)}}\left\langle v^{\prime}, \mu_{1}\left(\left(a_{0}^{(1)}, A^{(1)}\right), A^{(-1)}\right) v\right\rangle\right|_{A^{(-1)}, A^{(1)}=\mathbf{0}, a_{0}^{(1)}=1} \\
= & \sum_{k \in \mathbb{Z}_{+}}\left\langle v^{\prime}, z^{-k-2} L(-k) v\right\rangle+\left\langle v^{\prime}, z^{-2} L(0) v\right\rangle+\sum_{k \in \mathbb{Z}_{+}}\left\langle v^{\prime}, z^{k-2} L(k) v\right\rangle \\
= & \left.\left\langle v^{\prime}, \sum_{k \in \mathbb{Z}} L(k) x^{k-2} v\right\rangle\right|_{x=z}
\end{aligned}
$$

where the $L(k)$ 's are defined by

$$
\begin{gathered}
\left\langle v^{\prime}, L(k) v\right\rangle=-\left.\frac{\partial}{\partial A_{k}^{(1)}}\left\langle v^{\prime}, \mu_{1}\left(\left(a_{0}^{(1)}, A^{(1)}\right), A^{(-1)}\right) v\right\rangle\right|_{A^{(-1)}, A^{(1)}=\mathbf{0}, a_{0}^{(1)}=1}, \\
\left\langle v^{\prime}, L(0) v\right\rangle=-\left.\frac{\partial}{\partial a_{0}^{(1)}}\left\langle v^{\prime}, \mu_{1}\left(\left(a_{0}^{(1)}, A^{(1)}\right), A^{(-1)}\right) v\right\rangle\right|_{A^{(-1)}, A^{(1)}=\mathbf{0}, a_{0}^{(1)}=1}, \\
\left\langle v^{\prime}, L(-k) v\right\rangle=-\left.\frac{\partial}{\partial A_{k}^{(-1)}}\left\langle v^{\prime}, \mu_{1}\left(\left(a_{0}^{(1)}, A^{(1)}\right), A^{(-1)}\right) v\right\rangle\right|_{A^{(-1)}, A^{(1)}=\mathbf{0}, a_{0}^{(1)}=1}
\end{gathered}
$$

for $k>0$.

This geometric definition of the $L(k)$ operators is identical to the definition of the $L(k)$ operators in the vertex operator algebra setting of [H2]. Thus we may 
claim the proof from vertex operator algebras $([\mathrm{H2},(5.4 .26))$ that these operators satisfy the Virasoro relations with the bracket as defined in $\mathrm{H2}$.

6. Grading: If we assume that $v \in V_{(k)}$, then observe that by the definition of $L(0)$ and the GVOC grading axiom

$$
\begin{aligned}
\left\langle v^{\prime}, L(0) v\right\rangle & =\left.\left(-\frac{\partial}{\partial a_{0}^{(1)}}\left\langle v^{\prime}, \mu_{1}\left(\mathbf{0},\left(a_{0}, \mathbf{0}\right)\right) v\right\rangle\right)\right|_{a_{0}=1} \\
& =\left.\left(-\frac{\partial}{\partial a_{0}^{(1)}}\left(a_{0}^{(1)}\right)^{-k}\left\langle v^{\prime}, v\right\rangle\right)\right|_{a_{0}=1} \\
& =\left\langle v^{\prime}, k v\right\rangle .
\end{aligned}
$$

7. $L(1)$-Derivative:

$$
\begin{aligned}
& \left.\left\langle v^{\prime},\left(L(1) \otimes I d_{V}\right) X^{\mu}(x) v\right\rangle\right|_{x=z} \\
& =\left.\left(\frac{-\partial}{\partial z_{0}}\left\langle v^{\prime},\left(\mu_{1}\left(\mathbf{0},\left(1,\left(z_{0}, 0,0, \ldots\right)\right)\right) \otimes I d_{V}\right) X^{\mu}(x) v\right\rangle\right)\right|_{x=z, z_{0}=0} \\
& =\left.\left(\frac{-\partial}{\partial z_{0}}\left\langle v^{\prime}, \mu_{1}\left(\mathbf{0},\left(1,\left(z_{0}, 0,0, \ldots\right)\right)\right)_{1} *_{-1} \mu_{2}\left(\left(z^{-1} ; \mathbf{0},(1, \mathbf{0}),(1, \mathbf{0})\right)\right) v\right\rangle\right)\right|_{z_{0}=0} \\
& \left.=\left(\frac{-\partial}{\partial z_{0}}\left\langle v^{\prime}, \mu_{2}\left(\mathbf{0},\left(1,\left(z_{0}, 0,0, \ldots\right)\right)\right)_{1} \infty_{-1}\left(z^{-1} ; \mathbf{0},(1, \mathbf{0}),(1, \mathbf{0})\right)\right) v\right\rangle\right)\left.\right|_{z_{0}=0} \\
& =\left.\left(\frac{-\partial}{\partial z_{0}}\left\langle v^{\prime}, \mu_{2}\left(z-z_{0} ; \mathbf{0},(1, \mathbf{0}),(1, \mathbf{0})\right) v\right\rangle\right)\right|_{z_{0}=0} \\
& =\left.\left(\frac{-\partial}{\partial z_{0}}\left(\left.\left\langle v^{\prime}, X^{\mu}(x) v\right\rangle\right|_{x=z-z_{0}}\right)\right)\right|_{z_{0}=0} \\
& =\left.\left(\left.\left\langle v^{\prime}, \frac{-\partial}{\partial x} X^{\mu}(x) v\right\rangle\right|_{x=z-z_{0}} \frac{\partial}{\partial z_{0}}\left(z-z_{0}\right)\right)\right|_{z_{0}=0} \\
& =\left.\left\langle v^{\prime}, \frac{\partial}{\partial x} X^{\mu}(x) v\right\rangle\right|_{x=z}
\end{aligned}
$$

This completes the proof that all axioms hold for $\left(V, X^{\mu}, c^{\mu}, \rho^{\mu}\right)$.

5.2. A map from VOCs to GVOCs. We now construct a map from the category of vertex operator coalgebras to the category of geometric vertex operator coalgebras.

Let $(V, X, c, \rho)$ be a VOC of rank $d$. Let $\mu_{n}^{X}: K^{*}(n) \mapsto \mathcal{H}_{V}(1, n)$ be defined by 


$$
\begin{aligned}
& \left\langle v^{\prime}, \mu_{n}^{X}\left(z_{-n+1}^{-1}, \ldots, z_{-1}^{-1} ; A^{(-n)},\left(a_{0}^{(-n+1)}, A^{(-n+1)}\right), \ldots,\right.\right. \\
& \left.\left.\left(a_{0}^{(-1)}, A^{(-1)}\right),\left(a_{0}^{(1)}, A^{(1)}\right)\right) v\right\rangle \\
& =\iota_{1 \cdots n-1}^{-1}\left\langle v^{\prime},\left(\left(a_{0}^{(-1)}\right)^{-L(0)} e^{-\sum_{j \in \mathbb{Z}_{+}} A_{j}^{(-1)} L(-j)} \otimes \cdots \otimes\left(a_{0}^{(-n+1)}\right)^{-L(0)}\right.\right.
\end{aligned}
$$

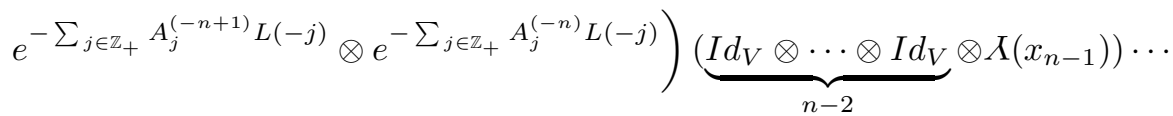

$$
\begin{aligned}
& \left.\left(I d_{V} \otimes X\left(x_{2}\right)\right) X\left(x_{1}\right) e^{-\sum_{j \in \mathbb{Z}_{+}} A_{j}^{(1)} L(j)}\left(a_{0}^{(1)}\right)^{-L(0)} v\right\rangle\left.\right|_{x_{i}=z_{-i}}
\end{aligned}
$$

for $n \in \mathbb{Z}_{+}$and

$$
\mu_{0}^{X}\left(\left(1, A^{(1)}\right)\right) v=c e^{-\sum_{j \in \mathbb{Z}_{+}} A_{j}^{(1)} L(j)} v
$$

for all $v \in V, v^{\prime} \in\left(V^{\otimes n}\right)^{\prime}$. The right-hand side of (5.3) is well defined since $v$ and $v^{\prime}$ are non-zero in on finitely many weights and degree raising operators are to the left of degree lowering operators.

At this point we need a lemma that gives us some information about absolute convergence when two sewings are composed. In and of itself this lemma says little because of its strong conditions, but it will be necessary in the general proof that $\left(V,\left\{\mu_{n}^{X}\right\}_{n \in \mathbb{Z}}\right)$ is a GVOC.

Lemma 5.2. Let $\ell, m \in \mathbb{N}$ and $n \in \mathbb{Z}_{+}$such that $m+n>1$. Choose $Q_{1} \in$ $K^{*}(\ell), Q_{2} \in K^{*}(m), Q_{3} \in K^{*}(n)$ and integers $1 \leq i \leq m$ and $1 \leq j \leq n$. Assume that the sewing $\left(Q_{1}{ }_{1} \infty_{-i} Q_{2}\right)_{1} \infty_{-j} Q_{3}$ exists. Then if, for all $v^{\prime} \in\left(V^{\otimes \ell+m+n-2}\right)^{\prime}$, $v \in V$,

$$
\left\langle v^{\prime}, \mu_{\ell+m-1}^{X}\left(Q_{1}{ }_{1} \infty_{-i} Q_{2}\right) v\right\rangle=\left\langle v^{\prime},\left(\mu_{\ell}^{X}\left(Q_{1}\right)_{1} *_{-i} \mu_{m}^{X}\left(Q_{2}\right)\right) v\right\rangle e^{\Gamma_{1} d}
$$

and

$$
\begin{aligned}
& \left\langle v^{\prime}, \mu_{\ell+m+n-2}^{X}\left(\left(Q_{11} \infty_{-i} Q_{2}\right)_{1} \infty_{-j} Q_{3}\right) v\right\rangle= \\
& \left\langle v^{\prime},\left(\mu_{\ell+m-1}^{X}\left(Q_{1}{ }_{1} \infty_{-i} Q_{2}\right)_{1} *_{-j} \mu_{n}^{X}\left(Q_{3}\right)\right) v\right\rangle e^{\Gamma_{2} d}
\end{aligned}
$$

for $\Gamma_{1}, \Gamma_{2}$ appropriately chosen, we have

$$
\begin{aligned}
& \left\langle v^{\prime}, \mu_{\ell+m+n-2}^{X}\left(\left(Q_{1}{ }_{1} \infty_{-i} Q_{2}\right)_{1} \infty_{-j} Q_{3}\right) v\right\rangle= \\
& \left\langle v^{\prime},\left(\left(\mu_{\ell}^{X}\left(Q_{1}\right)_{1 *_{-i}} \mu_{m}^{X}\left(Q_{2}\right)\right)_{1} *_{-j} \quad \mu_{n}^{X}\left(Q_{3}\right)\right) v\right\rangle e^{\left(\Gamma_{1}+\Gamma_{2}\right) d}
\end{aligned}
$$

and, in particular, the right-hand side of Equation 5.4 does exist.

Similarly, for $1 \leq i \leq m+n-1$ and $1 \leq j \leq n$, assume that the sewing $Q_{1}{ }_{1} \infty_{-i}\left(Q_{2}{ }_{1} \infty_{-j} Q_{3}\right)$ exists. If, for all $v^{\prime} \in\left(V^{\otimes \ell+m+n-2}\right)^{\prime}, v \in V$,

$$
\left\langle v^{\prime}, \mu_{m+n-1}^{X}\left(Q_{2}{ }_{1} \infty_{-j} Q_{3}\right) v\right\rangle=\left\langle v^{\prime},\left(\mu_{m}^{X}\left(Q_{2}\right)_{1} *_{-j} \mu_{n}^{X}\left(Q_{3}\right)\right) v\right\rangle e^{\Gamma_{1} d}
$$

and 


$$
\begin{aligned}
& \left\langle v^{\prime}, \mu_{\ell+m+n-2}^{X}\left(Q_{1}{ }_{1} \infty_{-i}\left(Q_{2}{ }_{1} \infty_{-j} Q_{3}\right)\right) v\right\rangle \\
& =\left\langle v^{\prime},\left(\mu_{\ell}^{X}\left(Q_{1}\right)_{1} *_{-i} \quad \mu_{m+n-1}^{X}\left(Q_{2}{ }_{1} \infty_{-j} Q_{3}\right)\right) v\right\rangle e^{\Gamma_{2} d}
\end{aligned}
$$

for $\Gamma_{1}, \Gamma_{2}$ appropriately chosen, we have

$$
\begin{aligned}
\left\langle v^{\prime}, \mu_{\ell+m+n-2}^{X}\right. & \left.\left(Q_{1}{ }_{1} \infty_{-i}\left(Q_{2}{ }_{1} \infty_{-j} Q_{3}\right)\right) v\right\rangle \\
& =\left\langle v^{\prime},\left(\mu_{\ell}^{X}\left(Q_{1}\right)_{1} *_{-i}\left(\mu_{m}^{X}\left(Q_{2}\right)_{1} *_{-j} \mu_{n}^{X}\left(Q_{3}\right)\right)\right) v\right\rangle e^{\left(\Gamma_{1}+\Gamma_{2}\right) d}
\end{aligned}
$$

and, in particular, the right-hand side of Equation 5.5 does exist.

Proof. This result uses the Fischer-Grauert Theorem (Theorem 3.4.3 in [H2]) and is essentially the double absolute convergence result given in part (5e) of Proposition 5.4.1 in [H2]. (It also follows Proposition 7.1 in [B] ). To prove the first half of the lemma, let $t_{1}, t_{2} \in \mathbb{C}^{\times}$, then multiply the local coordinate map at the $-i$-th puncture of $Q_{2}$ by $t_{1}$ and at the $-j$-th puncture of $Q_{3}$ by $t_{2}$ to obtain $Q_{2}\left(t_{1}\right) \in$ $K^{*}(m)$ and $Q_{3}\left(t_{2}\right) \in K^{*}(n)$, respectively. There exists a neighborhood of $(1,1) \in$ $\mathbb{C}^{\times} \times \mathbb{C}^{\times}$such that the sewing still exists. Using the definitions necessary to expand both sides of (5.4), we can observe that substituting $Q_{2}\left(t_{1}\right)$ and $Q_{3}\left(t_{2}\right)$ for $Q_{2}$ and $Q_{3}$ on the left-hand side of (5.4) shows that both sides are equal when viewed as formal power series in $t_{1}$ and $t_{2}$. (This substitution is valid because of the FischerGrauert Theorem.) Since the left-hand side is doubly absolutely convergent at $t_{1}=t_{2}=1$, so is the right-hand side.

The argument for the second half of the lemma is similar.

We now move to the main proposition of this section.

Proposition 5.3. The pair $\left(V,\left\{\mu_{n}^{X}\right\}_{n \in \mathbb{Z}}\right)$ is a geometric vertex operator coalgebra of rank $d$, where $d$ is the rank of the $\operatorname{VOC}(V, \Lambda, c, \rho)$.

Proof. Essentially, we use the axioms of a VOC to obtain the axioms of a GVOC:

1. Grading: Let $v^{\prime} \in V^{\prime}$ and $v \in V_{(k)}$. Then by the VOC grading axiom (4.6),

$$
\begin{aligned}
\left\langle v^{\prime}, \mu_{1}^{X}(\mathbf{0},(a, \mathbf{0})) v\right\rangle & =\left\langle v^{\prime}, a^{-L(0)} v\right\rangle \\
& =a^{-k}\left\langle v^{\prime}, v\right\rangle .
\end{aligned}
$$

2. Meromorphicity: From right rationality (Proposition4.5(i)) and the definition of $\mu_{n}^{X}$, we see that the map $Q \rightarrow\left\langle v^{\prime}, \mu_{n}^{X}(Q) v\right\rangle$ is meromorphic on $K(n)$. Fixing $v \in V$, we must show that for some $N(v) \in \mathbb{Z}_{+}$the degree of $z_{-i}$ is less than $N(v)$ in $\left\langle v^{\prime}, \mu_{n}^{X}(Q) v\right\rangle$ for any $1 \leq i \leq n-1$ and $v^{\prime} \in\left(V^{\otimes n}\right)^{\prime}$. We may assume $Q=\left(z_{-n+1}^{-1}, \ldots, z_{-1}^{-1} ; \mathbf{0},(1, \mathbf{0}), \ldots,(1, \mathbf{0})\right)$.

Considering repeated application of commutativity (Propositions 4.5 (iii)), we see that

$$
\begin{aligned}
& \left\langle v^{\prime}, \mu_{n}^{X}(Q) v\right\rangle \\
& \quad=\left.\iota_{1 \cdots n-1}^{-1}\left\langle v^{\prime},(\underbrace{I d_{V} \otimes \cdots \otimes I d_{V}}_{n-2} \otimes X\left(x_{n-1}\right)) \cdots\left(I d_{V} \otimes X\left(x_{2}\right)\right) X\left(x_{1}\right) v\right\rangle\right|_{x_{\ell}=z_{-\ell}}
\end{aligned}
$$




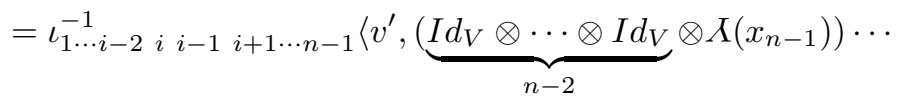

$$
\begin{aligned}
& (\underbrace{I d_{V} \otimes \cdots \otimes I d_{V}}_{i} \otimes X\left(x_{i+1}\right))(\underbrace{I d_{V} \otimes \cdots \otimes I d_{V}}_{i-2} \otimes\left(T \otimes I d_{V}\right)\left(I d_{V} \otimes X\left(x_{i-1}\right)\right) X\left(x_{i}\right)) \\
& \left.(\underbrace{I d_{V} \otimes \cdots \otimes I d_{V}}_{i-3} \otimes X\left(x_{i-2}\right)) \cdots\left(I d_{V} \otimes X\left(x_{2}\right)\right) X\left(x_{1}\right) v\right\rangle\left.\right|_{x_{\ell}=z_{-\ell}} \\
& =\iota_{i 1 \cdots \hat{i} \cdots n-1}^{-1}\left\langle v^{\prime},(\underbrace{I d_{V} \otimes \cdots \otimes I d_{V}}_{n-2} \otimes X\left(x_{n-1}\right)) \cdots\right. \\
& (\underbrace{I d_{V} \otimes \cdots \otimes I d_{V}}_{i} \otimes X\left(x_{i+1}\right))(\underbrace{I d_{V} \otimes \cdots \otimes I d_{V}}_{i-2} \otimes \\
& \left.\left(T \otimes I d_{V}\right)\left(I d_{V} \otimes X\left(x_{i-1}\right)\right) \cdots\left(T \otimes I d_{V}\right)\left(I d_{V} \otimes X\left(x_{1}\right)\right) X\left(x_{i}\right) v\right\rangle\left.\right|_{x_{\ell}=z_{-\ell}} .
\end{aligned}
$$

By the truncation axiom, there exists $N(v) \in \mathbb{Z}_{+}$such that the term $X\left(x_{i}\right) v$ has less than $N(v)$ positive powers of $x_{i}$. Thus we have less that $N(v)$ positive powers of $z_{-i}$.

3. Permutation: Given $Q \in K^{*}(n)$, we note that $S_{n}$ is generated by the actions of the transpositions $\sigma=(i i+1)$ for $i=1,2, \ldots, n-1$ so it suffices to show that the axiom holds for these. If $i<n-1$, the definition of the action and Proposition 4.5 (commutativity) suffice. The calculation is somewhat lengthy and found in Hub1]. If $i=n-1$, the argument requires not commutativity but the algebraic facts (4.8), (4.13), 4.21) and 4.15). Again, see Hub1 for the explicit calculation.

4. Sewing:

To prove the sewing axiom, consider $Q_{1} \in K^{*}(m)$ and $Q_{2} \in K^{*}(n)$ and $1 \leq i \leq$ $n$. Our proof will use the approach in $[\mathbb{B}$ and consider 8 steps. Step (a) will be the case that $m=0,1, n=1$, (b) - (f) will cover special cases needed for the induction, (g) is induction on $m$, and (h) is induction on $n$.

Throughout these steps, we will make use of the basis $\left\{e_{l^{(k)}}^{(k)} \mid l^{(k)}=1, \ldots, \operatorname{dim} V_{(k)}\right.$; $k \in \mathbb{Z}\}$ of $V^{\otimes m}$ and $\left\{\left(e_{l^{(k)}}^{(k)}\right)^{*} \mid l^{(k)}=1, \ldots, \operatorname{dim} V_{(k)} ; k \in \mathbb{Z}\right\}$ the corresponding dual basis.

Step (a): Since $K(1)=K^{*}(1)$, the case $Q_{1}, Q_{2} \in K^{*}(1)=K(1)$ where

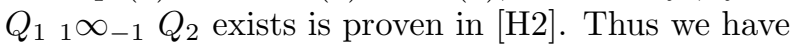

$$
\left\langle v^{\prime}, \mu_{1}^{X}\left(Q_{1}{ }_{1} \infty_{-1} Q_{2}\right) v\right\rangle=\left\langle v^{\prime},\left(\mu_{1}^{X}\left(Q_{1}\right)_{1} *_{-1} \mu_{1}^{X}\left(Q_{2}\right) v\right\rangle e^{-\Gamma\left(A^{(1)}, B^{(-1)}, a_{0}^{(1)}\right) d}\right.
$$

The case $Q_{1} \in K^{*}(0), Q_{2} \in K^{*}(1)$ is similar, but without needing $v^{\prime} \in V^{\prime}$.

Step (b): Now consider the case $i=2$ with

$$
\begin{gathered}
Q_{1}=\left(A^{(-1)},\left(a_{0}^{(1)}, A^{(1)}\right)\right) \in K^{*}(1), \\
Q_{2}=\left(\zeta^{-1} ; \mathbf{0},\left(b_{0}^{(-1)}, B^{(-1)}\right),(1, \mathbf{0})\right) \in K^{*}(2),
\end{gathered}
$$

such that $Q_{1}{ }_{1} \infty_{-i} Q_{2}$ exists.

$$
\left\langle v^{\prime},\left(\mu_{1}^{X}\left(Q_{1}\right)_{1} *_{-i} \mu_{2}^{X}\left(Q_{2}\right)\right)_{t} v\right\rangle
$$




$$
\begin{gathered}
=\sum_{k \in \mathbb{Z}} \sum_{l^{(k)}=1}^{\operatorname{dim} V_{(k)}}\left\langle v^{\prime},\left(I d_{V} \otimes\left(e^{-\sum_{j \in \mathbb{Z}_{+}} A_{j}^{(-1)} L(-j)} e^{-\sum_{j \in \mathbb{Z}_{+}} A_{j}^{(1)} L(j)}\left(a_{0}^{(1)}\right)^{-L(0)}\right)\right.\right. \\
\left.\left.e_{l^{(k)}}^{(k)}\left(e_{l^{(k)}}^{(k)}\right)^{*}\right)\left(\left(b_{0}^{(-1)}\right)^{-L(0)} e^{-\sum_{j \in \mathbb{Z}_{+}} B_{j}^{(-1)} L(-j)} \otimes I d_{V}\right) X(x) v\right\rangle\left.\right|_{x=\zeta} t^{k} \\
=\sum_{k \in \mathbb{Z}} \sum_{l^{(k)}=1}^{\operatorname{dim} V_{(k)}}\left\langle v^{\prime},\left(I d_{V} \otimes\left(e^{-\sum_{j \in \mathbb{Z}_{+}} A_{j}^{(-1)} L(-j)} e^{-\sum_{j \in \mathbb{Z}_{+}} A_{j}^{(1)} L(j)}\left(a_{0}^{(1)}\right)^{-L(0)} t^{L(0)}\right)\right.\right. \\
=\left.\left\langle v^{\prime},\left(I d_{V} \otimes e^{-\sum_{j \in \mathbb{Z}_{+}} A_{j}^{(-1)} L(-j)} e^{-\sum_{j \in \mathbb{Z}_{+}} A_{j}^{(k)} L(j)}\left(a_{0}^{(1)} t^{-1}\right)^{-L(0)}\right)\left(\left(b_{0}^{(-1)}\right)^{-L(0)} e^{-\sum_{j \in \mathbb{Z}_{+}} B_{j}^{(-1)} L(-j)} \otimes I d_{V}\right) X(x) v\right\rangle\right|_{x=\zeta} \\
\left.\left(\left(b_{0}^{(-1)}\right)^{-L(0)} e^{-\sum_{j \in \mathbb{Z}_{+}} B_{j}^{(-1)} L(-j)} \otimes I d_{V}\right) X(x) v\right\rangle\left.\right|_{x=\zeta} \\
=\left\langle v^{\prime},\left(\left(b_{0}^{(-1)}\right)^{-L(0)} e^{-\sum_{j \in \mathbb{Z}_{+}} B_{j}^{(-1)} L(-j)} \otimes e^{-\sum_{j \in \mathbb{Z}_{+}} A_{j}^{(-1)} L(-j)}\right)\right. \\
\left.\left.\left(I d_{V} \otimes e^{-\sum_{j \in \mathbb{Z}_{+}} A_{j}^{(1)} L(j)}\left(a_{0}^{(1)} t^{-1}\right)^{-L(0)}\right){ }^{-\sum_{j \in \mathbb{Z}_{+}} A_{j}^{(1)} L(j)}\left(a_{0}^{(1)} t^{-1}\right)^{-1}\right)^{-L(0)} v\right\rangle\left.\right|_{x=\zeta} .
\end{gathered}
$$

On the other hand, if we consider $Q_{2}$ as above and

$$
Q_{1}(t)=\left(A^{(-1)},\left(a_{0}^{(1)} t^{-1}, A^{(1)}\right)\right) \in K^{*}(1),
$$

then by Example 2.21 we observe that the image under $\mu_{2}^{X}$ of the sewing $Q_{1}(t)_{1} \infty_{-2} Q_{2}$ exists for $t=1$. Thus

$$
\begin{gathered}
\mu_{2}^{X}\left(Q_{1}(t){ }_{1} \infty_{-2} Q_{2}\right) \\
=\left\langle v^{\prime},\left(\left(b_{0}^{(-1)}\right)^{-L(0)} e^{-\sum_{j \in \mathbb{Z}_{+}} B_{j}^{(-1)} L(-j)}\left(a_{0}^{(1)} t^{-1}\right)^{L(0)} e^{-\Theta_{0}^{(2)} L(0)}\right.\right. \\
\left.e^{-\sum_{j \in \mathbb{Z}_{+}}\left(a_{0}^{(1)} t^{-1}\right)^{-j} \Theta_{j}^{(2)} L(-j)} \otimes e^{-\sum_{j \in \mathbb{Z}_{+}} A_{j}^{(-1)} L(-j)}\right) \\
\left.\quad X\left(a_{0}^{(1)} t^{-1} \hat{f}_{2}(x)\right) e^{-\sum_{j \in \mathbb{Z}_{+}} A_{j}^{(1)} L(j)}\left(a_{0}^{(1)} t^{-1}\right)^{-L(0)} v\right\rangle\left.\right|_{x=\zeta},
\end{gathered}
$$

where $\Theta_{j}^{(2)}$, for $j \in \mathbb{N}$, and $\hat{f}_{2}(x)$ are considered as functions of $a_{0}^{(1)}, A^{(1)}$ and $x$. It therefore remains to show that

$$
\begin{aligned}
& \text { 5.6) }\left(I d_{V} \otimes e^{-\sum_{j \in \mathbb{Z}_{+}} A_{j}^{(1)} L(j)}\left(a_{0}^{(1)} t^{-1}\right)^{-L(0)}\right) X(x)\left(a_{0}^{(1)} t^{-1}\right)^{L(0)} e^{\sum_{j \in \mathbb{Z}_{+}} A_{j}^{(1)} L(j)} \\
& =\left(\left(a_{0}^{(1)} t^{-1}\right)^{L(0)} e^{-\Theta_{0}^{(2)} L(0)} e^{-\sum_{j \in \mathbb{Z}_{+}}\left(a_{0}^{(1)} t^{-1}\right)^{-j} \Theta_{j}^{(2)} L(-j)} \otimes I d_{V}\right) X\left(a_{0}^{(1)} t^{-1} \hat{f}_{2}(x)\right) .
\end{aligned}
$$


Using (4.8), (4.22), and Proposition 3.4 we observe that the right-hand side of (5.6) is equal to

$$
\begin{gathered}
\left(\left(a_{0}^{(1)} t^{-1}\right)^{L(0)} e^{-\Theta_{0}^{(2)} L(0)} e^{-\sum_{j \in \mathbb{Z}_{+}}\left(a_{0}^{(1)} t^{-1}\right)^{-j} \Theta_{j}^{(2)} L(-j)} e^{a_{0}^{(1)} t^{-1}\left(-x+\hat{f}_{2}(x)\right) L(1)} \otimes I d_{V}\right) \\
X\left(a_{0}^{(1)} t^{-1} x\right) \\
=\left(e^{-\Theta_{0}^{(2)} L(0)} e^{-\sum_{j \in \mathbb{Z}_{+}} \Theta_{j}^{(2)} L(-j)} e^{\left(-x+\hat{f}_{2}(x)\right) L(1)}\left(a_{0}^{(1)} t^{-1}\right)^{L(0)} \otimes I d_{V}\right) X\left(a_{0}^{(1)} t^{-1} x\right) \\
=\left(e^{\sum_{k=-1}^{\infty}\left(\sum_{j \in \mathbb{Z}_{+}}\left(a_{0}^{(1)} t^{-1}\right)^{-j} A_{j}^{(1)}\left(\begin{array}{c}
-j+1 \\
k+1
\end{array}\right) x^{-j-k}\right) L(-k)}\left(a_{0}^{(1)} t^{-1}\right)^{L(0)} \otimes I d_{V}\right) X\left(a_{0}^{(1)} t^{-1} x\right) \\
=\left(\left(a_{0}^{(1)} t^{-1}\right)^{L(0)} \otimes I d_{V}\right)\left(e^{\sum_{k=-1}^{\infty}\left(\sum_{j \in \mathbb{Z}_{+}}\left(a_{0}^{(1)} t^{-1}\right)^{-j-k} A_{j}^{(1)}\left(\begin{array}{c}
-j+1 \\
k+1
\end{array}\right) x^{-j-k}\right) L(-k)} \otimes I d_{V}\right) \\
X\left(a_{0}^{(1)} t^{-1} x\right) .
\end{gathered}
$$

On the other hand, using (4.21), we see that the left-hand side of (5.6) is equal to

$$
\begin{aligned}
& \left(I d_{V} \otimes e^{-\sum_{j \in \mathbb{Z}_{+}} A_{j}^{(1)} L(j)}\right)\left(\left(a_{0}^{(1)} t^{-1}\right)^{L(0)} \otimes I d_{V}\right) X\left(a_{0}^{(1)} t^{-1} x\right) e^{\sum_{j \in \mathbb{Z}_{+}} A_{j}^{(1)} L(j)} \\
= & \left(\left(a_{0}^{(1)} t^{-1}\right)^{L(0)} \otimes I d_{V}\right)\left(I d_{V} \otimes e^{-\sum_{j \in \mathbb{Z}_{+}} A_{j}^{(1)} L(j)}\right) X\left(a_{0}^{(1)} t^{-1} x\right) e^{\sum_{j \in \mathbb{Z}_{+}} A_{j}^{(1)} L(j)} .
\end{aligned}
$$

Thus it suffices to show that

$$
\begin{array}{r}
\left(\sum_{k=-1}^{\infty}\left(\sum_{j \in \mathbb{Z}_{+}}\left(a_{0}^{(1)} t^{-1}\right)^{-j-k} A_{j}^{(1)}\left(\begin{array}{c}
-j+1 \\
k+1
\end{array}\right) x^{-j-k}\right) L(-k) \otimes I d_{V}\right) X\left(a_{0}^{(1)} t^{-1} x\right) \\
=X\left(a_{0}^{(1)} t^{-1} x\right) \sum_{j \in \mathbb{Z}_{+}} A_{j}^{(1)} L(j)-\left(I d_{V} \otimes \sum_{j \in \mathbb{Z}_{+}} A_{j}^{(1)} L(j)\right) X\left(a_{0}^{(1)} t^{-1} x\right) .
\end{array}
$$

Define

$$
h\left(x_{1}\right)=\sum_{j \in \mathbb{Z}_{+}} A_{j}^{(1)} x_{1}^{-j+1}
$$

and notice that

$$
\operatorname{Res}_{x_{1}}\left(h\left(x_{1}\right) \sum_{m \in \mathbb{Z}} L(m) x_{1}^{m-2}\right)=\sum_{j \in \mathbb{Z}_{+}} A_{j}^{(1)} L(j) .
$$

Now recalling (4.12) and observing that $h\left(x_{1}\right)$ commutes with all other terms, we observe that the right-hand side of (5.7) is 


$$
\begin{aligned}
& \operatorname{Res}_{x_{1}}\left(X\left(a_{0}^{(1)} t^{-1} x\right) h\left(x_{1}\right) \sum_{m \in \mathbb{Z}} L(m) x_{1}^{m-2}\right. \\
& \left.-\left(I d_{V} \otimes h\left(x_{1}\right) \sum_{m \in \mathbb{Z}} L(m) x_{1}^{m-2}\right) X\left(a_{0}^{(1)} t^{-1} x\right)\right) \\
& =\operatorname{Res}_{x_{1}} \operatorname{Res}_{x_{0}}\left(a_{0}^{(1)} t^{-1} x\right)^{-1} \delta\left(\frac{x_{1}-x_{0}}{a_{0}^{(1)} t^{-1} x}\right)\left(h\left(x_{1}\right) \sum_{m \in \mathbb{Z}} L(m) x_{0}^{m-2} \otimes I d_{V}\right) \\
& \Lambda\left(a_{0}^{(1)} t^{-1} x\right) \\
& =\operatorname{Res}_{x_{1}} \operatorname{Res}_{x_{0}} x_{1}^{-1} \delta\left(\frac{a_{0}^{(1)} t^{-1} x+x_{0}}{x_{1}}\right)\left(\sum_{j \in \mathbb{Z}_{+}} A_{j}^{(1)} x_{1}^{-j+1} \sum_{m \in \mathbb{Z}} L(m) x_{0}^{m-2} \otimes I d_{V}\right) \\
& \Lambda\left(a_{0}^{(1)} t^{-1} x\right) \\
& =\operatorname{Res}_{x_{0}}\left(\sum_{j \in \mathbb{Z}_{+}} A_{j}^{(1)}\left(a_{0}^{(1)} t^{-1} x+x\right)^{-j+1} \sum_{m \in \mathbb{Z}} L(m) x_{0}^{m-2} \otimes I d_{V}\right) X\left(a_{0}^{(1)} t^{-1} x\right) \\
& =\operatorname{Res}_{x_{0}}\left(\sum_{j \in \mathbb{Z}_{+}} A_{j}^{(1)} \sum_{k=-1}^{\infty}\left(\begin{array}{c}
-j+1 \\
k+1
\end{array}\right)\left(a_{0}^{(1)} t^{-1} x\right)^{-j-k} x_{0}^{k+1} \sum_{m \in \mathbb{Z}} L(m) x_{0}^{m-2} \otimes I d_{V}\right) \\
& \Lambda\left(a_{0}^{(1)} t^{-1} x\right) .
\end{aligned}
$$

But this is equal to the left-hand side of (5.7). Therefore, since $\mu_{2}^{X}\left(Q_{1}(t)_{1} \infty_{-2} Q_{2}\right)$ exists when $t=1$,

$$
\begin{aligned}
\mu_{2}^{X}\left(Q_{1}(1)_{1} \infty_{-2} Q_{2}\right) & =\left.\left\langle v^{\prime},\left(\mu_{1}^{X}\left(Q_{1}\right)_{1} *_{-2} \mu_{2}^{X}\left(Q_{2}\right)\right)_{t} v\right\rangle\right|_{t=1} \\
& =\left\langle v^{\prime},\left(\mu_{1}^{X}\left(Q_{1}\right)_{1} *_{-2} \mu_{2}^{X}\left(Q_{2}\right)\right) v\right\rangle .
\end{aligned}
$$

Step (c): Let $i=2$,

$$
\begin{gathered}
Q_{1}=\left(A^{(-1)},\left(a_{0}^{(1)}, A^{(1)}\right)\right) \in K^{*}(1), \\
Q_{2}=\left(\zeta^{-1} ; B^{(-2)},\left(b_{0}^{(-1)}, B^{(-1)}\right),(1, \mathbf{0})\right) \in K^{*}(2),
\end{gathered}
$$

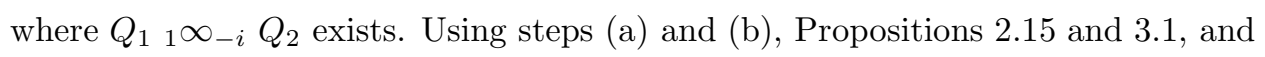
Lemma 5.2 


$$
\begin{aligned}
\mu_{2}^{X} & \left(Q_{1}{ }_{1} \infty_{-2} Q_{2}\right) \\
= & \mu_{2}^{X}\left(\left(Q_{1}{ }_{1} \infty_{-1}\left((1, \mathbf{0}), B^{(-2)}\right)\right)_{1} \infty_{-2}\left(\zeta^{-1} ;(1, \mathbf{0}),\left(b_{0}^{(-1)}, B^{(-1)}\right), \mathbf{0}\right)\right) \\
= & \mu_{1}^{X}\left(\left(Q_{1}\right)_{1} \infty_{-1}\left((1, \mathbf{0}), B^{(-2)}\right)\right)_{1} *_{-2} \mu_{2}^{X}\left(\left(\zeta^{-1} ;(1, \mathbf{0}),\left(b_{0}^{(-1)}, B^{(-1)}\right), \mathbf{0}\right)\right) \\
= & \left(\left(\mu_{1}^{X}\left(Q_{1}\right)_{1} *_{-1} \quad \mu_{1}^{X}\left(\left((1, \mathbf{0}), B^{(-2)}\right)\right)\right){ }_{1} *_{-2}\right. \\
& \left.\mu_{2}^{X}\left(\left(\zeta^{-1} ;(1, \mathbf{0}),\left(b_{0}^{(-1)}, B^{(-1)}\right), \mathbf{0}\right)\right)\right) e^{-\Gamma\left(A^{(1)}, B^{(-2)}, a_{0}^{(1)}\right) d} \\
= & \left(\mu _ { 1 } ^ { X } ( Q _ { 1 } ) _ { 1 } * _ { - 2 } \quad \left(\mu_{1}^{X}\left(\left((1, \mathbf{0}), B^{(-2)}\right)\right){ }_{1} *_{-2}\right.\right. \\
& \left.\left.\mu_{2}^{X}\left(\left(\zeta^{-1} ;(1, \mathbf{0}),\left(b_{0}^{(-1)}, B^{(-1)}\right), \mathbf{0}\right)\right)\right)\right) e^{-\Gamma\left(A^{(1)}, B^{(-2)}, a_{0}^{(1)}\right) d} \\
= & \left(\mu_{1}^{X}\left(Q_{1}\right)_{1} *_{-2} \mu_{2}^{X}\left(Q_{2}\right)\right) e^{-\Gamma\left(A^{(1)}, B^{(-2)}, a_{0}^{(1)}\right) d}
\end{aligned}
$$

Step (d): Let $i=1$,

$$
\begin{gathered}
Q_{1}=\left(A^{(-1)},\left(a_{0}^{(1)}, A^{(1)}\right)\right) \in K^{*}(1), \\
Q_{2}=\left(\zeta^{-1} ; B^{(-2)},\left(b_{0}^{(-1)}, B^{(-1)}\right),(1, \mathbf{0})\right) \in K^{*}(2),
\end{gathered}
$$

where $Q_{1}{ }_{1} \infty_{-i} Q_{2}$ exists.

Let $\sigma \in S_{2}$ be the transposition of two elements. We use the naturality of permutations (Propositions 2.16 and 3.2 and axiom 4), along with part (c) to observe the following:

$$
\begin{aligned}
& \mu_{2}^{X}\left(Q_{1}{ }_{1} \infty_{-1} Q_{2}\right)=\mu_{2}^{X}\left(\sigma\left(Q_{1}{ }_{1} \infty_{-2} \sigma Q_{2}\right)\right) \\
& =\sigma\left(\mu_{1}^{X}\left(Q_{1}\right)_{1} *_{-2} \quad \mu_{2}^{X}\left(\sigma Q_{2}\right)\right) e^{-\Gamma\left(A^{(1)}, B^{(-1)}\left(\left(b_{0}^{(-1)}\right)^{-1}\right), a_{0}^{(1)}\right) d} \\
& =\sigma\left(\mu_{1}^{X}\left(Q_{1}\right){ }_{1} *_{-2} \sigma \mu_{2}^{X}\left(Q_{2}\right)\right) e^{-\Gamma\left(A^{(1)}, B^{(-1)}\left(\left(b_{0}^{(-1)}\right)^{-1}\right), a_{0}^{(1)}\right) d} \\
& =\left(\mu_{1}^{X}\left(Q_{1}\right)_{1} *_{-1} \quad \mu_{2}^{X}\left(Q_{2}\right)\right) e^{-\Gamma\left(A^{(1)}, B^{(-1)}\left(\left(b_{0}^{(-1)}\right)^{-1}\right), a_{0}^{(1)}\right) d} .
\end{aligned}
$$

The careful reader will have noticed the inputs for $\Gamma$ are modified to $A^{(1)}$, $B^{(-1)}\left(\left(b_{0}^{(-1)}\right)^{-1}\right)=\left(\left(b_{0}^{(-1)}\right)^{-1} B_{1}^{(-1)},\left(b_{0}^{(-1)}\right)^{-2} B_{2}^{(-1)}, \ldots\right)$, and $a_{0}^{(1)}$ because we are working with $\sigma Q_{2}$ instead of $Q_{2}$. Via Proposition 4.2.1 in [H2], we have

$$
\Gamma\left(A^{(1)}, B^{(-1)}\left(\left(b_{0}^{(-1)}\right)^{-1}\right), a_{0}^{(1)}\right)=\Gamma\left(A^{(1)}, B^{(-1)}, a_{0}^{(1)} b_{0}^{(-1)}\right) .
$$

Step (e): Let $i=n$,

$$
\begin{gathered}
Q_{1}=\left(z^{-1} ; A^{(-2)},\left(a_{0}^{(-1)}, A^{(-1)}\right),(1, \mathbf{0})\right) \in K^{*}(2), \\
Q_{2}=\left(\zeta_{-n+1}^{-1}, \ldots, \zeta_{-1}^{-1} ; B^{(-n)},\left(b_{0}^{(-n+1)}, B^{(-n+1)}\right), \ldots,\right. \\
\left.\left.\left(b_{0}^{(-1)}, B^{(-1}\right)\right),\left(b_{0}^{(1)}, B^{(1)}\right)\right) \in K^{*}(n),
\end{gathered}
$$

where $Q_{1}{ }_{1} \infty_{-n} Q_{2}$ exists. Then the argument mimics that of Step (b) but with (2.28) replacing (2.26). (See Hub1 for details.)

Step (f): Let $1 \leq i \leq n$,

$$
Q_{1}=\left(z_{-1}^{-1} ; A^{(-2)},\left(a_{0}^{(-1)}, A^{(-1)}\right),(1, \mathbf{0})\right) \in K^{*}(2),
$$




$$
\begin{aligned}
Q_{2}=\left(\zeta_{-n+1}^{-1}, \ldots, \zeta_{-1}^{-1} ; B^{(-n)},\left(b_{0}^{(-n+1)}, B^{(-n+1)}\right), \ldots,\right. \\
\left.\left.\left(b_{0}^{(-1)}, B^{(-1}\right)\right),\left(b_{0}^{(1)}, B^{(1)}\right)\right) \in K^{*}(n),
\end{aligned}
$$

such that $Q_{1}{ }_{1} \infty_{-i} Q_{2}$ exists. If $\sigma$ is defined to be the transposition $(i n)$, then following the proof of Step (d), with $\tau$ the permutation $(i n+1 i+1 i+2 \ldots n)$,

$$
\begin{aligned}
\mu_{n+1}^{X}\left(Q_{1}{ }_{1} \infty_{-i} Q_{2}\right) & =\mu_{n+1}^{X}\left(\tau\left(Q_{1}{ }_{1} \infty_{-n} \sigma Q_{2}\right)\right) \\
& =\tau\left(\mu_{n+1}^{X}\left(Q_{1}{ }_{1} \infty_{-n} \sigma Q_{2}\right)\right) \\
& =\tau\left(\mu_{2}^{X}\left(Q_{1}\right)_{1} *_{-n} \mu_{n}^{X}\left(\sigma Q_{2}\right)\right) e^{\left.-\Gamma\left(A^{(1)}, B^{(-i)}\left(b_{0}^{(-i)}\right)^{-1}\right), a_{0}^{(1)}\right) d} \\
& =\tau\left(\mu_{2}^{X}\left(Q_{1}\right)_{1} *_{-n} \sigma \mu_{n}^{X}\left(Q_{2}\right)\right) e^{-\Gamma\left(A^{(1)}, B^{(-i)}, a_{0}^{(1)} b_{0}^{(-i)}\right) d} \\
& =\left(\mu_{2}^{X}\left(Q_{1}\right)_{1} *_{-i} \mu_{n}^{X}\left(Q_{2}\right)\right) e^{-\Gamma\left(A^{(1)}, B^{(-i)}, a_{0}^{(1)} b_{0}^{(-i)}\right) d}
\end{aligned}
$$

where Step (e) gives the key equality.

Step (g): We will now use induction on $m$ for $m \geq 2$. Assume that for all $\ell<m$ the sewing axiom holds and let $i \leq n$,

$$
\begin{aligned}
& Q_{1}=\left(z_{-m+1}^{-1}, \ldots, z_{-1}^{-1} ; A^{(-m)},\left(a_{0}^{(-m+1)},\right.\right.\left.A^{(-m+1)}\right), \ldots, \\
&\left.\left.\left(a_{0}^{(-1)}, A^{(-1}\right)\right),\left(a_{0}^{(1)}, A^{(1)}\right)\right) \in K^{*}(m), \\
& Q_{2}=\left(\zeta_{-n+1}^{-1}, \ldots, \zeta_{-1}^{-1} ; B^{(-n)},\left(b_{0}^{(-n+1)}, B^{(-n+1)}\right), \ldots,\right. \\
&\left.\left.\left(b_{0}^{(-1)}, B^{(-1}\right)\right),\left(b_{0}^{(1)}, B^{(1)}\right)\right) \in K^{*}(n),
\end{aligned}
$$

such that $Q_{1}{ }_{1} \infty_{-i} Q_{2}$ exists.

We begin by decomposing $Q_{1}$ into

$$
\begin{gathered}
\left.Q_{1}^{+}=\left(z_{-m+1}^{-1} ; A^{(-m)},\left(a_{0}^{(-m+1)}, A^{(-m+1}\right)\right),(1, \mathbf{0})\right), \\
\left.Q_{1}^{-}=\left(z_{-m+2}^{-1}, \ldots, z_{-1}^{-1} ; \mathbf{0},\left(a_{0}^{(-m+2)}, A^{(-m+2)}\right), \ldots,\left(a_{0}^{(-1)}, A^{(-1}\right)\right),\left(a_{0}^{(1)}, A^{(1)}\right)\right) .
\end{gathered}
$$

Then as in Step (c) (using associativity, the $t$-contraction and Lemma 5.2)

$$
\begin{aligned}
& \mu_{m+n-1}^{X}\left(Q_{1}{ }_{1} \infty_{-i} Q_{2}\right) \\
& =\mu_{m+n-1}^{X}\left(\left(Q_{1}^{+}{ }_{1} \infty_{-m+1} Q_{1}^{-}\right){ }_{1} \infty_{-i} Q_{2}\right) \\
& =\mu_{m+n-1}^{X}\left(Q_{1}^{+}{ }_{1} \infty_{-i-m+2}\left(Q_{1}^{-}{ }_{1} \infty_{-i} Q_{2}\right)\right) \\
& =\mu_{2}^{X}\left(Q_{1}^{+}\right)_{1} *_{-i-m+2} \mu_{m+n-2}^{X}\left(Q_{1}^{-}{ }_{1} \infty_{-i} Q_{2}\right) \\
& =\left(\mu_{2}^{X}\left(Q_{1}^{+}\right)_{1} *_{-i-m+2}\left(\mu_{m-1}^{X}\left(Q_{1}^{-}\right)_{1} *_{-i} \mu_{n}^{X}\left(Q_{2}\right)\right)\right) e^{-\Gamma\left(A^{(1)}, B^{(-i)}, a_{0}^{(1)} b_{0}^{(-i)}\right) d} \\
& =\left(\left(\mu_{2}^{X}\left(Q_{1}^{+}\right)_{1} *_{-m+1} \quad \mu_{m-1}^{X}\left(Q_{1}^{-}\right)\right)_{1} *_{-i} \mu_{n}^{X}\left(Q_{2}\right)\right) e^{-\Gamma\left(A^{(1)}, B^{(-i)}, a_{0}^{(1)} b_{0}^{(-i)}\right) d} \\
& =\left(\mu_{m}^{X}\left(Q_{1}\right)_{1} *_{-i} \quad \mu_{n}^{X}\left(Q_{2}\right)\right) e^{-\Gamma\left(A^{(1)}, B^{(-i)}, a_{0}^{(1)} b_{0}^{(-i)}\right) d}
\end{aligned}
$$

where the third, fourth and sixth equalities employ the inductive assumption and, in the case $m=2$, Step (f). 
Step (h): We will now use induction on $n$ for $n \geq 2$. Assume that for all $\ell<n$ the sewing axiom holds, let $i, Q_{1}$ and $Q_{2}$ be as in Step (h) and decompose $Q_{2}$ as

$$
\begin{gathered}
\left.Q_{2}^{+}=\left(\zeta_{-n+1}^{-1} ; B^{(-n)},\left(b_{0}^{(-n+1)}, B^{(-n+1}\right)\right),(1, \mathbf{0})\right), \\
\left.Q_{2}^{-}=\left(\zeta_{-1}^{-n+2}, \ldots, \zeta_{-1}^{-1} ; \mathbf{0},\left(b_{0}^{(-n+2)}, B^{(-n+2)}\right), \ldots,\left(b_{0}^{(-1)}, B^{(-1}\right)\right),\left(b_{0}^{(1)}, B^{(1)}\right)\right) .
\end{gathered}
$$

There are three possibilities:

(i) If $i=n$, then

$$
\begin{aligned}
& Q_{1{ }_{1} \infty_{-n}} Q_{2}=Q_{1{ }_{1} \infty_{-n}}\left(Q_{2}^{+}{ }_{1} \infty_{-n+1} Q_{2}^{-}\right) \\
& =\left(\begin{array}{lll}
Q_{1}{ }_{1} \infty_{-2} & Q_{2}^{+}
\end{array}\right)_{1} \infty_{-n+1} Q_{2}^{-} .
\end{aligned}
$$

For $n>2$, the sewing follows from the inductive assumption, associativity, the $t$-contraction and Lemma 5.2. For $n=2$, we supplement induction with the Steps (c) and (g).

(ii) If $i=n-1$, then

$$
\begin{aligned}
& Q_{1{ }_{1} \infty_{-n+1}} Q_{2}=Q_{1{ }_{1} \infty_{-n+1}}\left(Q_{2}^{+}{ }_{1} \infty_{-n+1} Q_{2}^{-}\right) \\
& =\left(\begin{array}{lll}
Q_{1}{ }_{1} \infty_{-1} Q_{2}^{+} & { }_{1} \infty_{-n+1} Q_{2}^{-}
\end{array}\right.
\end{aligned}
$$

For $n>2$, the sewing follows from the inductive assumption, associativity, the $t$-contraction and Lemma 5.2 For $n=2$, we supplement induction with the Steps (d) and (g).

(iii) For $i<n-1$, then

$$
\begin{aligned}
Q_{1{ }_{1} \infty_{-i} Q_{2}} & =Q_{1{ }_{1} \infty_{-i}}\left(Q_{2}^{+}{ }_{1} \infty_{-n+1} Q_{2}^{-}\right) \\
& =Q_{2}^{+}{ }_{1} \infty_{-m-n+2}\left(Q_{11} \infty_{-i} Q_{2}^{-}\right) .
\end{aligned}
$$

The sewing follows from the inductive assumption, Step (e), associativity, the $t$ contraction and Lemma 5.2

5.3. The categorical isomorphism between GVOAs and VOAs. Let $d$ be a complex number, $\mathbf{V}^{*}(d)$ be the category of vertex operator coalgebras of rank $d$, and $\mathbf{G}^{*}(d)$ be the category of geometric vertex operator coalgebras of rank $d$. In the previous two sections we have defined functors

$$
\begin{aligned}
F_{\mathbf{V}^{*}(d)}: \mathbf{V}^{*}(d) & \rightarrow \mathbf{G}^{*}(d) \\
(V, X, c, \rho) & \mapsto\left(V, \mu^{X}\right)
\end{aligned}
$$

and

$$
\begin{aligned}
F_{\mathbf{G}^{*}(d)}: \mathbf{G}^{*}(d) & \rightarrow \mathbf{V}^{*}(d) \\
(V, \mu) & \mapsto\left(V, X_{\mu}, c_{\mu}, \rho_{\mu}\right) .
\end{aligned}
$$

We have shown explicitly that these two maps take objects to objects and recalling our discussion of morphisms in the introduction of Section 5 it is evident that they respect morphisms as well (See also Hub1]). The main purpose of defining $F_{\mathbf{V}^{*}(d)}$ 
and $F_{\mathbf{G}^{*}(d)}$ is to show that they are inverses to each other and that the categories of VOCs and GVOCs are isomorphic.

Theorem 5.4. The categories $\mathbf{V}^{*}(d)$ and $\mathbf{G}^{*}(d)$ are isomorphic. In particular, the functors $F_{\mathbf{V}^{*}(d)}$ and $F_{\mathbf{G}^{*}(d)}$ satisfy

$$
\begin{aligned}
& F_{\mathbf{G}^{*}(d)} F_{\mathbf{V}^{*}(d)}=1_{\mathbf{V}^{*}(d)}, \\
& F_{\mathbf{V}^{*}(d)} F_{\mathbf{G}^{*}(d)}=1_{\mathbf{G}^{*}(d)}
\end{aligned}
$$

where $1_{\mathbf{V}^{*}(d)}$ and $1_{\mathbf{G}^{*}(d)}$ are the identity functors on $\mathbf{V}^{*}(d)$ and $\mathbf{G}^{*}(d)$, respectively.

Proof. Since the second half of the theorem implies the first half, we will simply prove (5.8) and (5.9). First, observe that

and

$$
c_{\mu}=\mu_{0}^{X}((1, \mathbf{0}))=c,
$$

$$
\rho_{\mu}=-\left.\frac{d}{d \epsilon} \mu_{0}^{X}(1,(0, \epsilon, 0,0, \ldots))\right|_{\epsilon=0}=-\left.\frac{d}{d \epsilon} c e^{-\epsilon L(2)}\right|_{\epsilon=0}=c L(2)=\rho
$$

by employing (4.17). For any $v^{\prime} \in V \otimes V$,

$$
\begin{aligned}
\operatorname{Res}_{x} x^{n}\left\langle v^{\prime}, X^{\mu}(x) v\right\rangle & =\operatorname{Res}_{z} z^{n}\left\langle v^{\prime}, \mu^{X}\left(\left(z^{-1} ;(1, \mathbf{0}),(1, \mathbf{0}), \mathbf{0}\right)\right)(v)\right\rangle \\
& =\operatorname{Res}_{z} z^{n}\left(\left.\left\langle v^{\prime}, X(x) v\right\rangle\right|_{x=z}\right) \\
& =\operatorname{Res}_{x} x^{n}\left\langle v^{\prime}, X(x) v\right\rangle .
\end{aligned}
$$

Thus (5.8) is verified.

As for (5.9), we need only verify that

$$
\begin{gathered}
\begin{aligned}
\left\langle v^{\prime}, \mu_{n}\left(z_{-1}^{-1}, \ldots, z_{-n+1}^{-1} ;\left(a_{0}^{(1)}, A^{(1)}\right),\left(a_{0}^{(-1)}, A^{(-1)}\right), \ldots,\right.\right. \\
\left.\left.\left(a_{0}^{(-n+1)}, A^{(-n+1)}\right), A^{(-n)}\right) v\right\rangle \\
=\iota_{x \cdots n-1}^{-1}\left\langle v^{\prime},\left(e^{-\sum_{j \in \mathbb{Z}_{+}} A_{j}^{(-1)} L(-j)}\left(a_{0}^{(-1)}\right)^{-L(0)} \otimes \cdots \otimes e^{-\sum_{j \in \mathbb{Z}_{+}} A_{j}^{(-n+1)} L(-j)}\right.\right. \\
\left.\left(a_{0}^{(-n+1)}\right)^{-L(0)} \otimes e^{-\sum_{j \in \mathbb{Z}_{+}} A_{j}^{(-n)} L(-j)}\right)(\underbrace{I d_{V} \otimes \cdots \otimes I d_{V}}_{n-2} \otimes X_{\mu}\left(x_{n-1}\right)) \cdots \\
\left.\left(I d_{V} \otimes X_{\mu}\left(x_{2}\right)\right) X_{\mu}\left(x_{1}\right) e^{-\sum_{j \in \mathbb{Z}_{+}} A_{j}^{(1)} L(j)}\left(a_{0}^{(1)}\right)^{-L(0)} v\right\rangle\left.\right|_{x_{i}=z_{-i}} .
\end{aligned}
\end{gathered}
$$

Since both sides of (5.10) define geometric vertex operator coalgebras, both satisfy the sewing axiom. Therefore, by Proposition 2.12 we need only prove (5.10) in the specialized cases

$$
\begin{gathered}
\left\langle v^{\prime}, \mu_{2}\left(z^{-1} ;(1, \mathbf{0}),(1, \mathbf{0}), \mathbf{0}\right) v\right\rangle=\left.\left\langle v^{\prime}, X_{\mu}(x) v\right\rangle\right|_{x=z}, \\
\mu_{0}((1, \mathbf{0})) v=c \\
\left\langle v^{\prime}, \mu_{1}\left(\left(a_{0}^{(1)}, A^{(1)}\right), A^{(-1)}\right) v\right\rangle=\left\langle v^{\prime}, e^{-\sum_{j \in \mathbb{Z}_{+}} A_{j}^{(-1)} L(-j)} e^{-\sum_{j \in \mathbb{Z}_{+}} A_{j}^{(1)} L(j)}\left(a_{0}^{(1)}\right)^{-L(0)} v\right\rangle .
\end{gathered}
$$


The first 2 of these equations are true by the definition of $F_{\mathbf{G}^{*}(d)}$. The third equation, the proof for $K^{*}(1)$, is identical to the proof for $K(1)$ in [H2] (Equation (5.4.31)) since $K(1)=K^{*}(1)$ in the most specific sense, the definitions of $L(0)$ are the same and the meromorphicity axiom is the same in that special case.

\section{REFERENCES}

[A] Lars Ahlfors, Conformal invariants: Topics in Geometric Function Theory, McGraw-Hill, New York, 1973.

[B] Katrina Barron The Notion of $N=1$ Supergeometric Vertex Operator Superalgebra and the Isomorphism Theorem, Communications in Contemporary Mathematics, Vol. 5, No. 4 (2003), 481-567.

[BHL] Katrina Barron, Yi-Zhi Huang, James Lepowsky, Factorization of formal exponentials and uniformization, Journal of Algebra 228 (2000), 551-579.

[BPZ] Alexander Belavin, A. E. Polyakov, Alexander Zamolodchikov, Infinite conformal symmetries in two-dimensional quantum field theory, Nuclear Physics, B241 (1984), 333-380.

[Bo] Richard Borcherds, Vertex algebras, Kac-Moody algebras, and the Monster, Proceedings of the National Academy of Science USA 83 (1986), 3068-3071.

[D] Chongying Dong, Introduction to vertex operator algebras I, Surikaisekikenkyusho Kokyuroku 904 (1995), 1-25.

[FK] Hershel M. Farkas and Irwin Kra, Riemann Surfaces, Second addition, Graduate Texts in Mathematics, 71, Springer-Verlag, New York, 1992.

[FHL] Igor B. Frankel, Yi-Zhi Huang, James Lepowsky, On Axiomatic Approaches to Vertex Operator Algebras and Modules, Memoirs of the AMS, Number 494, Providence, Rhode Island, July 1993.

[FS] Daniel Friedan, Stephen Shenker, The analytic geometry of two-dimensional conformal field theory, Nuclear Physics B281 (1987), 509-545.

[G] H. Guo, On abelian intertwining algebras and modules, Ph. D. thesis, Rutgers University, 1994.

[H1] Yi-Zhi Huang, On the geometric interpretation of vertex operator algebras, Ph.D. thesis, Rutgers University, 1990.

[H2] Yi-Zhi Huang Two-Dimensional Conformal Geometry and Vertex Operator Algebras, Birkhäuser, Boston, 1997.

[HL] Yi-Zhi Huang, James Lepowsky, Operadic formulation of the notion of vertex operator algebra, Contemporary Mathematics 175 (1994), 131-148.

[Hub1] Keith Hubbard, The notion of a vertex operator coalgebra: a construction and geometric characterization, Ph.D. thesis, University of Notre Dame, expected 2005.

[Hub2] Keith Hubbard, Constructions of vertex operator coalgebras via vertex operator algebras, preprint, arXiv:math.QA/0405461

[Hub3] Keith Hubbard, Vertex coalgebras, modules, commutativity and associativity, in preparation.

[FLM] Igor Frenkel, James Lepowsky, Arne Meurman, Vertex Operator Algebras and the Monster, Academic Press, Inc., San Diego, 1988.

[LL] James Lepowsky, Haisheng Li, Introduction to Vertex Operator Algebras and Their Representations, Birkhäuser, Boston, 2004.

[LW] James Lepowsky, Robert L. Wilson, Construction of the affine Lie algebra $A_{1}^{(1)}$, Communications in Mathematical Physics 62 (1978), 43-53.

[M] J. Peter May, The geometry of iterated loop spaces, Lecture Notes in Mathematics, Number 271, Springer-Verlag, 1972.

[MSS] Martin Markl, Steve Shnider, Jim Stasheff, Operads in Algebra, Topology and Physics, Mathematical Surverys and Monographs, Volume 96, American Mathematical Society, 2002.

[S] Graeme Segal, The definition of conformal field theory, preprint, 1988.

[V] Cumrun Vafa, Conformal theories and punctured surfaces, Phys. Lett. B199 (1987), 195-202. 